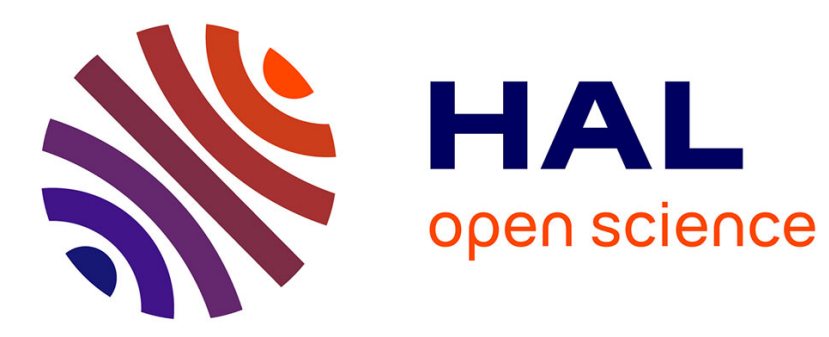

\title{
Mathematical model for transport of DNA plasmids from the external medium up to the nucleus by electroporation
}

Michael S Leguèbe, Maria Grazia Notarangelo, Monika Twarogowska, Roberto Natalini, Clair Poignard

\section{To cite this version:}

Michael S Leguèbe, Maria Grazia Notarangelo, Monika Twarogowska, Roberto Natalini, Clair Poignard. Mathematical model for transport of DNA plasmids from the external medium up to the nucleus by electroporation. Mathematical Biosciences, 2017, 285, pp.1-13. 10.1016/j.mbs.2016.11.015 . hal-01412380

\section{HAL Id: hal-01412380 \\ https://hal.inria.fr/hal-01412380}

Submitted on 8 Dec 2016

HAL is a multi-disciplinary open access archive for the deposit and dissemination of scientific research documents, whether they are published or not. The documents may come from teaching and research institutions in France or abroad, or from public or private research centers.
L'archive ouverte pluridisciplinaire HAL, est destinée au dépôt et à la diffusion de documents scientifiques de niveau recherche, publiés ou non, émanant des établissements d'enseignement et de recherche français ou étrangers, des laboratoires publics ou privés. 


\title{
Mathematical model for transport of DNA plasmids from the external medium up to the nucleus by electroporation
}

\author{
M. Leguèbe ${ }^{\mathrm{a}}$, M.G. Notarangelo ${ }^{\mathrm{b}}$, M. Twarogowska ${ }^{\mathrm{c}}$, R. Natalini ${ }^{\mathrm{b}}$, C. Poignard $^{\mathrm{d}}$ \\ ${ }^{a}$ Max-Planck-Institut für Sonnensystemforschung, Justus-von-Liebig-Weg 3, 3707r Göttingen, Germany. \\ ${ }^{b}$ Istituto per le Applicazioni del Calcolo "M. Picone", Consiglio Nazionale delle Ricerche, Via dei Taurini 19, I-00185 \\ Rome, Italy \\ ${ }^{c}$ Dipartimento di Ingegneria e Scienze dell'Informazione e Matematica, Universita degli Studi dell'Aquila, Via Vetoio, \\ Coppito, 67100 L'Aquila, Italy \\ ${ }^{d}$ Team MONC, INRIA Bordeaux-Sud-Ouest, Institut de Mathématiques de Bordeaux, CNRS UMR 5251 G Université \\ de Bordeaux, 351 cours de la Libération, 33405 Talence Cedex, France.
}

\begin{abstract}
We propose a mathematical model for the transport of DNA plasmids from the extracellular matrix up to the cell nucleus. The model couples two phenomena: the electroporation process, describing the cell membrane permeabilization to plasmids and the intracellular transport enhanced by the presence of microtubules. Numerical simulations of cells with arbitrary geometry, in 2D and 3D, and a network of microtubules show numerically the importance of the microtubules and the electroporation on the effectiveness of the DNA transfection, as observed by previous biological data. The paper proposes efficient numerical tools for forthcoming optimized procedures of cell transfection.

Keywords: Mathematical Biology, Intracellular Transport, Microtubules, Plasmids Transport, Finite Difference Method in Irregular Domains
\end{abstract}

\section{Introduction}

Plasmid DNA vaccination combined with electroporation (EP) provides a promising approach for the prevention of infectious diseases and for cancer immunotherapy. This technology generates an immune response due to an enhancement in expression of specific encoded antigens [27]. The use of pulsed electric field to vectorize the plasmid presents an efficient biophysical tool for DNA transfection without healthy risk as for viral gene transfection [27. The aim of the paper is to propose a model that describes in vitro plasmid transfection by mean of pulsed electric field, from the outer medium -the solution where cells are in suspension- up to the nucleus.

\subsection{Biological principles of gene electrotransfection}

DNA plasmids are extrachromosomal genetic materials engineered so that cells directly produce specific antigens to stimulate the immunological response. To be effective, it is necessary that plasmids reach the cell nucleus. However, due to their large size $(5-20 \mathrm{~kb})$, plasmids cannot cross the cell

Email address: clair.poignard@inria.fr (C. Poignard) 
membrane, and thus even for in vitro experiments, cells embedded in a bath saturated with a large amount of DNA plasmids do not acquire the new genetic material. In vivo, the situation is even worse since the extracellular matrix 1 provides another barrier before reaching the cell. As a consequence, most of DNA plasmids are degraded before entering the nucleus.

To overcome this drawbacks one non-viral possibility is to apply pulsed electric field on the site of injection to electropermeabilize transiently cell membranes and make them porous to DNA plasmids. More precisely, the effect of the electric pulse is twofold. On one hand, it increases the permeability of cytoplasmic membrane and facilitates the passage of the plasmids through it. Biological experiments demonstrate that macromolecules with molecular weight above $4 \mathrm{kDa}$, such as DNA plamids, cannot pass across the cytoplasmic membrane in normal conditions. A direct transfer to the cytoplasm is only observed if macromolecules are close to the membrane during the electric pulse delivery [12]. On the other hand, the electric field has an electrophoretic effect, which transports charged molecules such as DNA plasmid in the direction of the electric field [25] in the extracellular medium.

Inside the cell cytoplasm, plasmids transport is a combination of very slow free diffusion and an active transport along microtubules, which can be seen as cytoplasmic highways towards the nucleus. Recent studies demonstrate that while the cytoskeleton acts as a barrier to DNA plasmids movement in the cytoplasm, the microtubule network is required for plasmid trafficking directed towards the nucleus [2, 25]. In the absence of microtubules DNA plasmids do not arrive near the nucleus. However to be bound to microtubules and transported along it, plasmids need cytoplasmic adapter proteins such as molecular motors (dynein), transcriptional factors (TF), and importins. Once plasmids arrive at the end of the microtubules (close to the nucleus), they are released and aggregate around nucleus. Their large size does not permit the free passage across the nuclear pore complexes, which are present on the nuclear envelope. It is thus necessary that the DNA plasmids interact with importins which widen diameters of nuclear pore complexes.

\subsection{Need of numerical models}

From the biological point of view, the phenomenon is thus quite well understood, however, the literature suffers from a lack of numerical models describing the plasmid transport, from the extracellular medium up to the nucleus by mean of electroporation. The aim of this paper is to propose a model that addresses such a transport, which is a coupling between passive (diffusion) and active (electrophoretic and chemical along the microtubules) transports. On one hand we model the passage of DNA plasmids from extracellular matrix into the cell cytoplasm thanks to the effect of the pulsed electric field. On the other hand, we describe the intracellular traffic of plasmids enhanced by a network of microtubules, from the cytoplasm towards the nucleus. Since the electric field is almost null inside the cell due to the membrane shielding [7, 24, 33, we focus on two types of plasmid transport inside the cell (a slow free diffusion and the active transport along the microtubules). It is worth noting that the complete modeling of DNA uptake by mean of electroporation has never been addressed before. It is however crucial to obtain a reliable model with as less as parameter as possible in order to optimize

\footnotetext{
${ }^{1}$ Let us mention that cells growing in vitro also produce ECM in the Petri dish, however for the transfection, cells are usually washed out, trypsinised, and then resuspended, therefore one can consider that no ECM is present around the cell during the DNA transfection.
} 
cell transfection. The goal of the paper is to provide a first step towards such a numerical optimization of DNA uptake by mean of electroporating pulses.

Many mathematical models of intracellular trafficking developed in the last ten years concern micromolecules and are in terms of ordinary differential equations (ODEs). In (see [28, 13]) models without spatial localization of reactions were considered. Spatial dependence of intracellular transport was introduced in [20, 19, 26, 29, 11, 10, 31, 32, 8. Recent developments have been obtained by Cangiani et al. [3] and Dimitrio et al. [9]. Cangiani et al. combined a model of nuclear import pathway mediated by the RAN gradient with microtubules activity described by a vector field, while the work of Dimitrio et al. analyzed the effect of microtubules on the small protein p53. However no DNA uptake was considered. In particular, the role of microtubule, which is less important for small molecules which can diffuse freely in the cytoplasm, is crucial for large molecules as DNA plasmids. The role of cytoskeleton in intracellular DNA transport has been addressed in [22] (one can refer also to the PhD thesis [23]), but without electroporation, and the numerical framework was simplistic (a 2D-rectangular cell shape with one microtubule). Large plasmids are considered and equations for the transport along microtubules with RAN cycle are derived to account for the traffic of DNA in the cytoplasm, through the nuclear envelope, and its accumulation in the nucleus. Differently to Cangiani's approach, the microtubules are one-dimensional fibers to which plasmids can attach and are transported along towards nucleus. The attention was focused on the description of transport mechanisms in the whole cell and on interactions of plasmids with other molecules such as dynein, importin, and RAN cycle. However no realistic numerical simulations have been presented before. The goal of the paper is to combine the model of cell electroporation with the DNA transport inside the cell, and to provide a numerical method that makes it possible to simulate the complete model in realistic $2 \mathrm{D}-$ and $3 \mathrm{D}$ - configurations. Let us note also that optimization of cell transfection is the long-term goal of the project, it is thus important to propose reliable models with as less parameter as possible, and accurate numerical methods for the calibration of the model with the experiments.

\subsection{Outline of the paper}

In this paper we consider a simplified spatio-temporal model for intracellular transport proposed in [23] but in a more complex domain and we couple it with an electroporation model inspired from Leguèbe et al. [18]. More than just a combination of two existing models, we provide simplified models with less parameters, for forthcoming calibration of the model with experimental data. Even though the calibration is far from the scope of this paper, it is the long-term goal of this research. Therefore it is important to propose reliable and simple models, with accurate numerical schemes for the simulations: these are the two goals of the paper. Our purpose in this article is to present a simple model based on partial differential equations (PDE), which can account for the main phenomena involved in the plasmid transport, from the extracellular bath up to the nucleus, in order to highlight the influences of cell electropulsation on one side and the microtubule network on the other side.

The paper is organized as follows. In Section 2 is devoted to a precise description of our model, which is organised in 2 parts: the electrical part that describes how the membrane conductance and permeability are affected, and then the transport of plasmid in the cell. The main feature of the intracellular plasmid transport lies in the links between the spatial movement (free diffusion in 3D) and the 1D-transport along the microtubules. Then, in Section 3 we present the numerical scheme used to solve our PDE model and in particular to preserve the mass conservation. We validate our method 
by numerical considerations. We conclude by numerical simulations that corroborate experimental results in Section 4. Interestingly, our numerical tool makes it possible to compare simulations and experimental data, for instance these of Rosazza et al. [25]. To our best knowledge, it is the first time that such DNA uptake by mean of electroporation is described by PDE model, accounting for the spatial distribution of plasmids in the cell, from the extracellular bath up to the cell nucleus. Calibration of the model with experimental data are planned in forthcoming works.

\section{Presentation of the model}

This section is devoted to the presentation of our mathematical model for the transport of DNA plasmids from the extracellular medium up to the cell nucleus. The model is split into two parts: a system describing the electric field, which permits the plasmids to pass from the extracellular medium to the cell cytoplasm, and the model of the transport of plasmids inside the cell.

The electric pulses permeabilize cell membrane, allowing molecules that usually cannot cross the membrane barrier to enter in the cytoplasm. Moreover, plasmids are charged molecules and are subjected to electrophoretic forces, so the electric field enhances their transport in the extracellular matrix. Once plasmids reach the cell cytoplasm, they are trapped in the cytoskeleton and bind with importin and dynein molecules. The latter, through specific molecular pathways, make possible the attachment to microtubules and active transport along them. The DNA alone, that is without cytoplasmic adapter proteins, can only diffuse weakly in the cytoplasm.

Here we couple a simplified version of Leguèbe, Poignard et al. [15, 18, model for the electropermeabilization and a simplified Notarangelo and Natalini system for intracellular transport of plasmids [23].

First the geometrical setting of our problem is presented and the definition of the microtubules network, along which the cargo DNA+dynein+importin is transported, is detailed. Then, the description of the model of the transport of plasmids across the cell membrane and in the cytoplasm coupled with the electropermeabilization model is given. Throughout this article the following conventions and notations are used.

Notation 2.1. For any bounded domain $\mathcal{O}$ of $\mathbb{R}^{3}, \partial \mathcal{O}$ denotes its boundary. For a close surface without boundary $\gamma, \nu$ denotes its unitary normal vector directed outwardly from the inside to the outside of the domain enclosed by $\gamma$.

- The restriction to $\gamma$ of a function $f$ defined in the vicinity of $\gamma$ is denoted by $\left.f\right|_{\gamma}$. If $f$ is discontinuous across $\gamma$, then $\left.f\right|_{\gamma^{-}}\left(\right.$resp. $\left.\left.f\right|_{\gamma^{+}}\right)$denotes its restriction taken in the domain enclosed by $\gamma$ (resp. the outer domain).

- The jump of a function $f$ across the interface $\gamma$ is defined as:

$$
[f]_{\gamma}=\left.f\right|_{\gamma^{+}}-\left.f\right|_{\gamma^{-}}
$$

- The flux across $\gamma$ of a (smooth enough) function $f$ defined in the vicinity of $\gamma$ is defined by

$$
\left.\partial_{\nu} f\right|_{\gamma}=\left.\nabla f\right|_{\gamma} \cdot \nu
$$




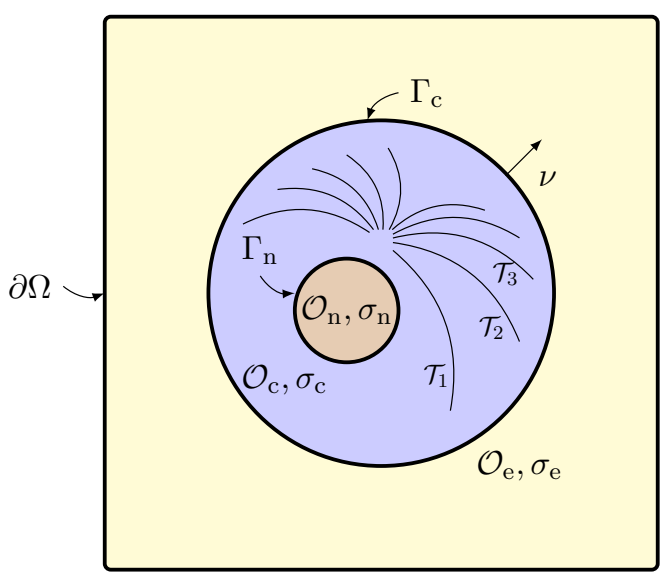

(a) Geometry of the whole domain

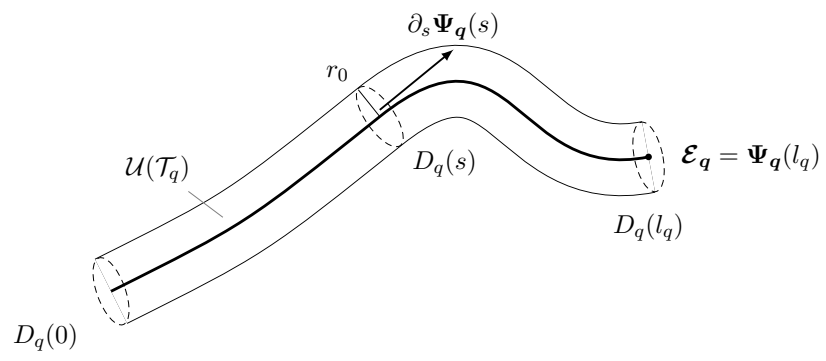

(b) Geometry of the microtubule

Figure 1: Geometrical settings. Figure 1(a) The nucleus is denoted by $\mathcal{O}_{\mathrm{n}}$, the cell cytoplasm is $\mathcal{O}_{\mathrm{c}}$ and the extracellular medium is $\mathcal{O}_{\mathrm{e}}$. The cell membrane is denoted by $\Gamma_{\mathrm{c}}$ while the nucleus membrane is $\Gamma_{\mathrm{n}}$. For the electric potential, homogeneous Neumann boundary conditions are imposed on $\Gamma_{\mathrm{N}}$, while Dirichlet source is imposed on $\Gamma_{\mathrm{D}}$. Figure $1(\mathrm{~b})$ The area of attraction $\mathcal{U}\left(\mathcal{T}_{q}\right)$ around the microtubule $\mathcal{T}_{q}$ in $3 \mathrm{D}$. For the sake of clarity, the radius $r_{0}$ has been considerably augmented compared to the microtubule length. According to [25], the ratio $\ell_{q} / r_{0}$ takes values between 100 and 1000 .

- Throughout the paper, $\beta$ stands for a regularised even Heaviside function in the form

$$
\beta(\lambda, \mu)=e^{-(\lambda / \mu)^{2}},
$$

but other sigmoidal functions could be used.

\subsection{Geometrical setting}

2.1.1. The extracellular domain, the cell cytoplasm and the cell nucleus.

We devide a smooth domain $\Omega \subset \mathbb{R}^{3}$ in three smooth subdomains: the extracellular medium $\mathcal{O}_{\mathrm{e}}$, and a cell composed of the nucleus $\mathcal{O}_{\mathrm{n}}$ and the cytoplasm $\mathcal{O}_{\mathrm{c}}$, as presented in Figure 1(a). The boundaries between each subdomains are supposed to be smooth, and

$$
\Gamma_{\mathrm{n}}=\partial \mathcal{O}_{\mathrm{n}}, \quad \Gamma_{\mathrm{c}}=\partial \mathcal{O}_{\mathrm{c}} \backslash \Gamma_{\mathrm{n}} .
$$

The outer boundary $\partial \Omega$ is split into two disjoint parts $\Gamma_{\mathrm{D}}$ and $\Gamma_{\mathrm{N}}$ on which Dirichlet (resp. Neumann) boundary conditions are imposed (see Fig. 1(a)):

$$
\partial \Omega=\Gamma_{\mathrm{D}} \cup \Gamma_{\mathrm{N}}, \quad \Gamma_{\mathrm{D}} \cap \Gamma_{\mathrm{N}}=\emptyset .
$$

\subsubsection{The microtubule network.}

We define microtubules as a finite network $\mathcal{T}$ of $N_{\mathcal{T}}$ segments $\left(\mathcal{T}_{q}\right)_{q=1}^{N_{\mathcal{T}}}$ in the cytoplasm between the vicinity of $\Gamma_{\mathrm{c}}$ and $\Gamma_{\mathrm{n}}$. Each microtubule is a 1D-curve parameterized thanks to its curvilinear abscissa (see Figure 1(b):

$$
\mathcal{T}_{q}:=\left\{\Psi_{q}(s), \forall s \in\left[0, \ell_{q}\right]\right\},
$$


where $\ell_{q}$ is the length of the microtubule $\mathcal{T}_{q}$. The orientation of the curvilinear abscissa is chosen so as the beginning of the microtubule $\Psi_{q}(s=0)$ is located near the cell membrane, while the ending point $\mathcal{E}_{q}$ is close to the nucleus

$$
\mathcal{E}_{q}=\Psi_{q}\left(\ell_{q}\right)
$$

\subsubsection{Area of attraction around the microtubules}

To each point $\Psi_{q}(s)$ of the microtubule $\mathcal{T}_{q}$ is associated a disk $D_{q}(s)$, of radius $r_{0}$ and perpendicular to the microtubule ${ }^{2}$ such that $\forall q=0,1, \cdots, N_{\mathcal{T}}, \forall s \in\left[0, \ell_{q}\right]$ it is defined as (see Figure 1(b))

$$
D_{q}(s):=\left\{\boldsymbol{\Psi}_{\boldsymbol{q}}(s)+\theta_{1} \boldsymbol{\nu}_{\mathbf{1}}+\theta_{2} \boldsymbol{\nu}_{\mathbf{2}}, \sqrt{\theta_{1}^{2}+\theta_{2}^{2}} \leqslant r_{0}, \boldsymbol{\nu}_{\mathbf{1}} \times \boldsymbol{\nu}_{\mathbf{2}}=\partial_{s} \boldsymbol{\Psi}_{\boldsymbol{q}}, \boldsymbol{\nu}_{\mathbf{1}} \perp \boldsymbol{\nu}_{\mathbf{2}},\left\|\boldsymbol{\nu}_{\mathbf{1}}\right\|=\left\|\boldsymbol{\nu}_{\mathbf{2}}\right\|=1\right\} .
$$

A tubular neighborhood $\mathcal{U}\left(\mathcal{T}_{q}\right)$ of radius $r_{0}$ is defined around each microtubule $\mathcal{T}_{q}$ (see Fig. 1(b)):

$$
\forall q=0,1, \cdots, N_{\mathcal{T}}, \quad \mathcal{U}\left(\mathcal{T}_{q}\right)=\left\{\Psi_{q}(s)+\eta(s), \quad \forall s \in\left(0, \ell_{q}\right), \forall \eta(s) \in D_{q}(s)\right\} .
$$

This neighborhood represents the attraction zone where the cargo DNA+dynein+importin bind to microtubules. Out of this region, the cargo cannot attach to it and the active transport does not occur.

\subsection{Model of electropermeabilization}

When submitted to high electric pulses, the cell membrane permeability increases and large molecules, which usually cannot diffuse through membrane, enter the cytoplasm. This phenomenon is called the electropermeabilization. In this section we present a simplified system of this process, derived by Leguèbe, Poignard et al. [15, 18. It consists of the Laplace equation for the potential in a cell and the particular transmission conditions on the cellular and nuclear membranes. The advantage of the following model is that it accounts for the main phenomena explained in [18], with less parameters, which is important for the fitting with experimental data.

\subsubsection{Cell Membrane conductance.}

Electroporation phenomena consists of an increase of cell membrane conductance due to a high transmembrane voltage, that is the difference of the electrical potential across the membrane. The electrical cell membrane conductance $S_{\mathrm{c}}$ depends non-linearly on the transmembrane voltage. Denoting by $V_{\Gamma_{\mathrm{c}}}$ this transmembrane voltage, we set the following non-linear law of membrane conductance 3 .

$$
S_{\mathrm{c}}\left(t, V_{\Gamma_{\Gamma_{\mathrm{c}}}}\right)=S_{0}+S_{1} \beta\left(V_{\mathrm{ep}}, V_{\left.\right|_{\Gamma_{\mathrm{c}}}}\right)+S_{2} X_{2}\left(t, V_{\left.\right|_{\Gamma_{\mathrm{c}}}}\right),
$$

where $S_{0}$ is the cell membrane conductance at rest, $S_{1}$ is the conductance of the permeabilized membrane during the pulse, $S_{2}$ is the long-term increased conductance after the pulse, with $S_{1} \gg S_{2} \geq S_{0}$,

\footnotetext{
${ }^{2}$ Note that in two dimensions, the disk $D(s)$ is nothing but segment of length $2 r_{0}$ perpendicular to the microtubule at the point $\Psi_{q}(s)$.

${ }^{3}$ Note that by definition of the function $\beta$ given by (1), the increase of the cell membrane conductance is related to the membrane electrostatic energy equal to $\frac{1}{2} C_{\mathrm{c}} V_{\left.\right|_{\Gamma_{\mathrm{c}}}}^{2}$.
} 
and $V_{\text {ep }}$ is the threshold value of the transmembrane voltage above which electroporation occurs. $S_{2}$ is the long-term increase of the membrane conductance, and $X_{2}$ is the degree of permeabilization, which describes the alteration of membrane lipids, leading to an increase of membrane permeability to the plasmids. It satisfies the following ordinary differential equation:

$$
\left\{\begin{array}{l}
X_{2}(t=0)=0, \\
\partial_{t} X_{2}=\max \left(\frac{\beta\left(V_{\mathrm{perm}}, V_{\left.\right|_{\Gamma_{\mathrm{c}}}}\right)-X_{2}}{\tau^{-}}, \frac{\beta\left(V_{\mathrm{perm}}, V_{\left.\right|_{\Gamma_{\mathrm{c}}}}\right)-X_{2}}{\tau^{+}}\right), \quad \forall t>0,
\end{array}\right.
$$

where $\tau^{+}$and $\tau^{-}$are the characteristic times of respectively the increase and the decrease of the degree of electropermeabilization, and $V_{\text {perm }}$ is the threshold of membrane voltage above which permeabilisation occurs. We refer to [18] and references therein for a precise description.

The membrane conductance $S_{\mathrm{c}}$ given by (2) is dynamical model of nonlinear membrane conductivity, which involves two phenomena: the pore formation during the pulse, and the alteration of membrane lipids after the pulse. This second phenomenon was not accounted for in [21, 16] or [15]. Note that the pore formation is accounted for by a static law (the term $S_{1} \beta$ in equation (2)), which is in accordance with Ivorra et al. [14. $X_{2}$ holds for the long-term effect of electroporation on membrane conductance, which has been experimentally observed by Wegner et al. [34. At the same time, Leguèbe et al. proposed a complex model to account for lipid alteration, that was reported by L.M. Mir's group, however the model involves many non-measurable parameters. We propose here a simplified version, with less parameters, of the model of Leguèbe et al. [18]. Since the electric field is very low inside the cell, no induced electroporation occurs, and thus the conductance of the nuclear membrane $S_{\mathrm{n}}$ is constant.

\subsubsection{Model of the electric potential}

The electrical conductivity chart of the domain $\Omega$ is defined as

$$
\sigma=\left\{\begin{array}{l}
\sigma_{\mathrm{e}} \text { in } \mathcal{O}_{\mathrm{e}} \\
\sigma_{\mathrm{c}} \text { in } \mathcal{O}_{\mathrm{c}} \\
\sigma_{\mathrm{n}} \text { in } \mathcal{O}_{\mathrm{n}}
\end{array}\right.
$$

where $\sigma_{\mathrm{e}}, \sigma_{\mathrm{c}}$ and $\sigma_{\mathrm{n}}$ are the conductivities of the extracellular medium, the cytoplasm and the nucleus respectively. The electrical properties of the membranes $\Gamma_{\mathrm{c}}$ and $\Gamma_{\mathrm{n}}$ are characterized by the couple surface capacitance and surface conductance denoted by $\left(C_{\mathrm{c}}, S_{\mathrm{c}}\right)$ and $\left(C_{\mathrm{n}}, S_{\mathrm{n}}\right)$ respectively for $\Gamma_{\mathrm{c}}$ and $\Gamma_{\mathrm{n}}$.

Following [15, 18] the electrical potential $U$ satisfies the following quasistatic problem:

$$
\begin{aligned}
\Delta U & =0 & \text { in } \mathcal{O}_{\mathrm{e}} \cup \mathcal{O}_{\mathrm{c}} \cup \mathcal{O}_{\mathrm{n}}, t>0 \\
U & =g(t) & \text { on } \Gamma_{\mathrm{D}}, t>0 \\
\partial_{\boldsymbol{\nu}} U & =0 & \text { on } \Gamma_{\mathrm{N}}, t>0 \\
U(0, \cdot) & =0, & \text { in } \mathcal{O}_{\mathrm{e}} \cup \mathcal{O}_{\mathrm{c}} \cup \mathcal{O}_{\mathrm{n}},
\end{aligned}
$$

where the Dirichlet data $g=g(t)$ is the time-dependent boundary condition on $\Gamma_{\mathrm{D}}$ determined by the 
pulse delivered to the cell, and the transmission conditions across $\Gamma_{\mathrm{c}}, \Gamma_{\mathrm{n}}$ are

$$
\begin{array}{ll}
\left.\sigma_{\mathrm{e}} \partial_{\nu} U\right|_{\Gamma_{\mathrm{c}}^{+}}=\left.\sigma_{\mathrm{c}} \partial_{\nu} U\right|_{\Gamma_{\mathrm{c}}^{-}}, & C_{\mathrm{c}} \partial_{t}[U]_{\Gamma_{\mathrm{c}}}+S_{\mathrm{c}}\left([U]_{\Gamma_{\mathrm{c}}}\right)[U]_{\Gamma_{\mathrm{c}}}=\left.\sigma_{\mathrm{c}} \partial_{\nu} U\right|_{\Gamma_{\mathrm{c}}^{-}}, \\
\left.\sigma_{\mathrm{c}} \partial_{\nu} U\right|_{\Gamma_{\mathrm{n}}^{+}}=\left.\sigma_{\mathrm{n}} \partial_{\nu} U\right|_{\Gamma_{\mathrm{n}}^{-}}, & C_{\mathrm{n}} \partial_{t}[U]_{\Gamma_{\mathrm{n}}}+S_{\mathrm{n}}[U]_{\Gamma_{\mathrm{n}}}=\left.\sigma_{\mathrm{n}} \partial_{\nu} U\right|_{\Gamma_{\mathrm{n}}^{-}} .
\end{array}
$$

The following well-posedness result is proven in the Appendix section.

Theorem 2.2 (Well-posedness of the electrical model). Let $g$ belong to $W^{1,1}\left((0, T) ; H^{1}\left(\partial \Omega_{\mathrm{D}}\right)\right)$, then there exists a unique solution $U$ to problem (4) which belongs to

$$
U \in C\left((0, T) ; P H^{3 / 2}(\Omega)\right) \cap C^{1}\left((0, T) ; P H^{1 / 2}(\Omega)\right),
$$

where

$$
P H^{s}(\Omega)=\left\{u \in L^{2}(\Omega):\left.u\right|_{\omega} \in H^{s}(\omega) \text { for } \omega \in\left\{\mathcal{O}_{\mathrm{e}}, \mathcal{O}_{\mathrm{c}}, \mathcal{O}_{\mathrm{n}}\right\}\right\}
$$

\subsection{Model of transport of DNA plasmids}

The aim of this section is to describe the model of the transport of DNA plasmids in the extracellular matrix and inside the cell. We consider two types of plasmids: the DNA alone, denoted by $M$, which is present in both extracellular $\mathcal{O}_{\mathrm{e}}$ and cytoplasmic $\mathcal{O}_{\mathrm{c}}$ domains, and the cargo $M^{*}$ composed of DNA+importin+dynein, which lives only in the cytoplasm $\mathcal{O}_{c}$. It is assumed that as soon as the plasmids $M$ enter the cell, they bind simultaneously to importin and dynein at a constant rate $k^{*}$.

Both molecules, $M$ and $M^{*}$, diffuse with the piecewise constant diffusion coefficients $d$ and $d^{*}$ respectively given as

$$
d=\left\{\begin{array}{l}
d_{\mathrm{e}} \text { in } \mathcal{O}_{\mathrm{e}}, \\
d_{\mathrm{c}} \text { in } \mathcal{O}_{\mathrm{c}}, \\
d_{\mathrm{n}} \text { in } \mathcal{O}_{\mathrm{n}},
\end{array} \quad d^{*}=\left\{\begin{array}{l}
d_{\mathrm{c}}^{*} \text { in } \mathcal{O}_{\mathrm{c}}, \\
d_{\mathrm{n}}^{*} \text { in } \mathcal{O}_{\mathrm{n}} .
\end{array}\right.\right.
$$

We emphasize that in the bath, the diffusion coefficient of $d_{\mathrm{e}}$ is much higher than the inner diffusion coefficents, due to the cytoplasmic materials, which prevent the free diffusion. Since DNA are charged molecules, they are subject to the electric forces. In the model the electrical pulses have double effect. They transport the plasmids $M$ in the extracellular matrix and they permit them to cross the cell's membrane by increasing its permeabilization. A model without the influence of the electric field was studied in [23. We note that the electrophoretic forces act only in the extracellular matrix. It is a plausible assumption because the electric field in the cytoplasm is very low due to the shielding effect of the membrane. Moreover, the cytoplasm is composed of cytoskeleton and organelles, which prevents large molecules from free motion. Additionally, thanks to the importin and dynein, the plasmids $M^{*}$ bind to microtubules at rate $k_{\mathcal{T}}$ and are actively transport along them with velocity $v_{\mathcal{T}}$ towards nucleus.

\subsubsection{Membrane permeability}

We assume that the membrane permeability increases due to the effect of the electric field, see [18]. Two phenomena are considered. In parallel to the high increase of the membrane permeability during the pulse, which is a result of the creation of pores, there is a long lasting effect of electropermeabilization characterized by the degree of permeabilization $X_{2}$. The total membrane permeability $P_{\mathrm{c}}$ is modeled as a sum of these two effects

$$
P_{\mathrm{c}}\left(t, V_{\Gamma_{\Gamma_{\mathrm{c}}}}\right)=P_{1} \beta\left(V_{\mathrm{ep}}, V_{\Gamma_{\Gamma_{\mathrm{c}}}}\right)+P_{2} X_{2}\left(t, V_{\Gamma_{\Gamma_{\mathrm{c}}}}\right), \quad \text { with } P_{1} \gg P_{2}>0 .
$$




\subsubsection{Transport of free $D N A$}

Plasmids $M$ without importin and dynein diffuse in the extracellular matrix and in the cell cytoplasm and are transported by the electrophoretic forces. Inside a cell they are degraded by enzymes at the rate $k_{\text {deg }}$ and bind with dynein and importin at the rate $k^{*}$ forming a complex denoted by $M^{*}$. They cannot be uptaken in the nucleus for which importin is required. Therefore, the homogeneous Neumann boundary conditions for $M$ on $\Gamma_{\mathrm{n}}$ are imposed. On the contrary, the cell membrane $\Gamma_{\mathrm{c}}$ is an interface with permeability $P_{\mathrm{c}}$ to DNA and we assume that the transmission across it satisfies the Kedem-Katachalsky conditions (6f).

The concentration of plasmids $M$ is modeled by the following reaction-diffusion system:

$$
\begin{array}{lc}
\partial_{t} M-d_{\mathrm{e}} \Delta M+\mu_{\mathrm{e}} \nabla \cdot(M \nabla U)=0, & \text { in } \mathcal{O}_{\mathrm{e}}, \\
\partial_{t} M-d_{\mathrm{c}} \Delta M=-k^{*} M-k_{\operatorname{deg}} M, & \text { in } \mathcal{O}_{\mathrm{c}}, \\
M(0, \cdot)=M^{0}, & \text { in } \mathcal{O}_{\mathrm{e}} \\
M=M^{0}, & \text { on } \partial \Omega,
\end{array}
$$

with the following transmission conditions at the interfaces $\Gamma_{\mathrm{c}}$ and $\Gamma_{\mathrm{n}}$ :

$$
\begin{aligned}
& \left.d_{\mathrm{e}} \partial_{\nu} M\right|_{\Gamma_{\mathrm{c}}^{+}}-\left.\mu_{\mathrm{e}} M \partial_{\nu} U\right|_{\Gamma_{\mathrm{c}}^{+}}=\left.d_{\mathrm{c}} \partial_{\nu} M\right|_{\Gamma_{\mathrm{c}}^{-}}, \\
& P_{\mathrm{c}}\left(t,[U]_{\Gamma_{\mathrm{c}}}\right)[M]_{\Gamma_{\mathrm{c}}}=\left.d_{\mathrm{c}} \partial_{\nu} M\right|_{\Gamma_{\mathrm{c}}^{-}}, \\
& \left.\partial_{\boldsymbol{\nu}} M\right|_{\Gamma_{\mathrm{n}}^{+}}=0 .
\end{aligned}
$$

\subsubsection{Transport of DNA-dynein-importin complex}

Plasmids $M$ after entering a cell bind to dynein and importin with rate $k^{*}$ and form a complex $M^{*}$. The molecules $M^{*}$ cannot leave the cell but, contrary to free plasmids $M$, can enter the nucleus. They diffuse, and when they are in the area of attraction of microtubules $\mathcal{U}(\mathcal{T})$, they bind to them at the rate $k_{\mathcal{T}}$ and are transported with speed $v_{\mathcal{T}}$ towards the nucleus. Plasmids attached to the $\mathcal{T}_{q}$ microtubule are denoted by $W_{q}$. The detachment from microtubules occurs at the ending point $\mathcal{E}_{q}$ located near the nucleus. The concentration of $M^{*}$ in the cell satisfies

$$
\begin{array}{lr}
\partial_{t} M^{*}-d_{\mathrm{c}}^{*} \Delta M^{*}=k^{*} M-k_{\mathrm{deg}}^{*} M^{*}-k_{\mathcal{T}} M^{*} \mathbb{1}_{\mathcal{U}(\mathcal{T})}+\frac{v_{\mathcal{T}}}{|D|} \sum_{q=1}^{N_{\mathcal{T}}} W_{q}\left(\mathcal{E}_{q}\right) \delta_{D\left(\mathcal{E}_{q}\right)}(x) & \text { in } \mathcal{O}_{\mathrm{c}}, \\
\partial_{t} M^{*}-d_{\mathrm{n}}^{*} \Delta M^{*}=0 & \text { in } \mathcal{O}_{\mathrm{n}}, \\
\left.\partial_{\boldsymbol{\nu}} M^{*}\right|_{\Gamma_{\mathrm{c}}^{-}}=0, & \text { in } \mathcal{O}_{\mathrm{c}} \cup \mathcal{O}_{\mathrm{n}} \\
M^{*}(0, \cdot)=0, &
\end{array}
$$

with the following transmission conditions on $\Gamma_{\mathrm{n}}$ :

$$
\begin{aligned}
& \left.d_{\mathrm{c}} \partial_{\nu} M^{*}\right|_{\Gamma_{\mathrm{n}}^{+}}=\left.d_{\mathrm{n}} \partial_{\nu} M^{*}\right|_{\Gamma_{\mathrm{n}}^{-}} \\
& P_{\mathrm{n}}\left[M^{*}\right] \Gamma_{\Gamma_{\mathrm{n}}}=\left.d_{\mathrm{n}} \partial_{\nu} M^{*}\right|_{\Gamma_{\mathrm{n}}^{-}},
\end{aligned}
$$


where $P_{N}$ is the nuclear membrane permeability, which is assumed not to be affected by the electric field.

Note that the factor $|D|$ in the last term in $(7 \mathrm{a})$, which models the total amount of cargo $W_{q}$ released to the cell cytoplasm at the end of the microtubules, accounts for the flux of the section ${ }^{4}$ of the attraction area at the ending point of the microtubule $\mathcal{E}_{q}$.

The cargo $W_{q}$ is transported along the microtubule $\mathcal{T}_{q}$ with velocity $v_{\mathcal{T}}$ according to

$$
\begin{aligned}
& \partial_{t} W_{q}(t, s)-v_{\mathcal{T}} \partial_{s} W_{q}(t, s)=k_{\mathcal{T}} \int_{D(s)} M^{*}(t, \tau) \mathrm{d} \tau, \quad \forall t>0, \forall s \in\left(0, \ell_{q}\right), \\
& W_{q}(t, s=0)=0, \quad W_{q}(0, \cdot)=0 .
\end{aligned}
$$

The last term describes the quantity of cargo $M^{*}$ that bounds to a microtubule. Note that $M^{*}$ is integrated on the cross section of the $\mathcal{U}\left(\mathcal{T}_{q}\right)$, i.e. on the disk $D$. Therefore the unit of each $W_{q}$ is homogeneous to the unit of $M^{*}$ times $\mathrm{m}^{2}$. In order to prevent plasmids from attaching continuously at the end of microtubules, the coefficient $k_{\mathcal{T}}$ is smoothly set to 0 in the vicinity of the nucleus on the last micrometer of the microtubules.

\subsubsection{Well-posedness and the total mass conservation}

There is no feedback from the transport model towards the electric model, and the transport equations are linear. As a consequence, the well-posedness of the transport equations follows from classical arguments. Additionally, in the absence of the degradation in the cytoplasm, the boundary conditions imply the mass conservation of the DNA that entered the cell cytoplasm.

Proposition 2.3. Let $\left(M, M^{*},\left(W_{q}\right)_{q=1, \cdots, N_{\mathcal{T}}}\right)$ be the solution to problem (6)-(7)-(8), with $k_{\text {deg }}=$ $k_{\text {deg }}^{*}=0$. Then the following mass conservation in a cell holds:

$$
\forall t>0, \quad \frac{d}{d t}\left(\int_{\mathcal{O}_{\mathrm{c}} \cup \mathcal{O}_{\mathrm{n}}} M(\cdot, x)+M^{*}(\cdot, x) d x+\sum_{q=1}^{N_{\mathcal{T}}} \int_{\mathcal{T}_{q}} W_{q}(\cdot, s) d s\right)=\int_{\Gamma_{\mathrm{c}}} d_{\mathrm{c}} \partial_{\boldsymbol{\nu}} M(t, x) d x
$$

Proof. The equality is a consequence of an integration of (6)-(7)-(8) and the application of Green formula.

\section{Numerical approximation}

This section focuses on the numerical methods used to solve the model presented in section 2, The scheme for the electrical part (4) of the model has been presented in [15] and extensively studied in [17]. Roughly speaking, it is a finite difference method on a Cartesian mesh adapted from the second order scheme of Cisternino and Weynans [4] and introduced in [17. The transport part, that is the reactiondiffusion systems (6), (7) and the linear advection equation (8), are approximated by a classical finite difference method in space and explicit-implicit first order time integration.

\footnotetext{
${ }^{4}$ Note that $|D|=\pi r_{0}^{2}$ since we have considered a cylindrical tubular neighborhood of radius $r_{0}$ (see Fig. 1(b)
} 
Numerical difficulties lie in the approximation of the attachment-detachment of plasmids to microtubules. At the discrete level the transition from the volumetric (cell cytoplasm) to the linear (microtubules) transport and vice-versa is not trivial. If a one dimensional grid of microtubules coincides exactly with the mesh nodes of the two-dimensional cytoplasmic domain, then the computation of the mass plasmids in the area of attraction uses directly the values of the mesh, see [23]. However, in our model the microtubules have arbitrary shape and localization. They don't necessarily coincide with the mesh grid; therefore interpolation methods are required. In this section we aim at describing the numerical approximations of the attachment-detachment processes that preserve the total mass of plasmids.

Note that the model in section 2 can be defined on a two- or tri-dimensional domain with onedimensional microtubules. For computational reasons, the simulations in section 4 are performed in a two-dimensional setting, and thus the following description of the numerical methods is done also in two space dimensions to simplify the notations.

We work on a Cartesian computational grid $\Omega_{h}$ of the domain $\Omega=(0, L)^{2}$. Each direction is discretized uniformly by $J$ points and characterized by the positive parameter $h$. The nodes are given by $\mathbf{x}_{i, j}=\left(x_{i}, y_{j}\right)=(i h, j h)$, for $(i, j) \in J^{2}$. The $q$-th microtubule after parametrization is discretized into $k_{\max }^{q}$ nodes uniformly distributed with spacing $\Delta s$. In what follows, when only one microtubule is considered we omitt the index $q$ in order to simplify the notation.

\subsection{Approximation of attachment-detachment of plasmids to microtubules}

\subsubsection{Attachment of plasmids to microtubules}

The location of microtubules does not coincide with the two dimensional mesh points and therefore the attachment of cargo $M^{*}$ to the microtubules in the area of attraction, described by $-k_{\mathcal{T}} M^{*} \mathbb{1}_{\mathcal{U}(\mathcal{T})}$

in (7a) and $k_{\mathcal{T}} \int_{D(s)} M^{*} \mathrm{~d} x$ in $8 \mathrm{a}$, requires the interpolation over the grid nodes. The total mass of plasmids $M^{*}$ in a section $D(s)$ of the area of attraction $\mathcal{U}(\mathcal{T})$ of a microtubule is given by

$$
I(t, s)=\int_{D(s)} M^{*}(t, x) \mathrm{d} x .
$$

Depending on the size of the section $D(s)$ with respect to the mesh grid, see Figure 2, the integral (9) for each node $s_{k}, k=1, \ldots, k_{\max }$ of the microtubule can be approximated by a single value

$$
I\left(t, s_{k}\right) \sim|D| M^{*}\left(t, p_{k}\right)
$$

where $p_{k}:=\Psi\left(s_{k}\right)$ is a point in $\Omega$, or by the trapezoidal rule (or any other quadrature)

$$
I\left(t, s_{k}\right) \approx \frac{|D|}{2 m_{\max }} \sum_{m=1}^{m_{\max }} M^{*}\left(t, p_{k}^{m}\right),
$$

where $p_{k}^{m}:=\Psi\left(s_{k}^{m}\right)$ and $m_{\max }$ is the fixed number of discretization points on $D\left(s_{k}\right)$. Note that the concentration $M^{*}$ is computed on the Cartesian grid, while points $p_{k}^{m}$ don't necessarily belong to it. 
In order to evaluate $M^{*}\left(p_{k}^{m}\right)$ a standard interpolation from the values of the mesh is performed, that is for any point $p_{k}^{m}$

$$
M^{*}\left(t, p_{k}^{m}\right) \approx \sum_{\alpha_{k}^{m}} b_{\alpha_{k}^{m}} M_{\alpha_{k}^{m}}^{*}
$$

where $\alpha_{k}^{m}$ is a 2-uple of indices denoting the Cartesian grid points neighboring the point $p_{k}^{m}$ and $M_{\alpha_{k}^{m}}^{*}$ are values of $M^{*}$ at these points, as presented in Figure 2 .

The following proposition describes the conditions on the interpolation coefficients $b_{\alpha_{k}^{m}}$ that assure the mass conservation during the attachment of plasmids to the microtubule. For simplicity, we consider that only one point is used to approximate the integral (9).

Proposition 3.1. Let the total mass on a disc $D\left(s_{k}\right), k=1, \ldots, k_{\max }$ by given by (10), and the total mass in the area of attraction writes as

$$
\int_{\mathcal{O}_{\mathrm{c}}} M^{*}(t, x) \mathbb{1}_{\mathcal{U}(\mathcal{T})} \mathrm{d} x=\sum_{\mathbf{x}_{i, j} \in \Omega_{h}} c_{i, j} M^{*}\left(\mathbf{x}_{i, j}\right) h^{2},
$$

where $M^{*}\left(\mathbf{x}_{i, j}\right)$ is the density of cargo at the node $\mathbf{x}_{i, j}$ and $c_{i, j}$ are the coefficients of the matrix approximating the characteristic function $\mathbb{1}_{\mathcal{U}(\mathcal{T})}$ on the mesh $\Omega_{h}$.

The total mass during the attachment of plasmids to microtubules is conserved if

$$
\sum_{\mathbf{x}_{i, j} \in \Omega_{h}} c_{i, j} M^{*}\left(\mathbf{x}_{i, j}\right) h^{2}=|D| \sum_{k=0}^{k_{\max }} \sum_{\alpha_{k}} b_{\alpha_{k}} M_{\alpha_{k}}^{*} \Delta s
$$

Proof. Considering the one dimensional domain of the microtubule the total mass of $M^{*}$ inside its area of attraction is given by

$$
\int_{\mathcal{O}_{\mathrm{c}}} M^{*}(t, x) \mathbb{1}_{\mathcal{U}(\mathcal{T})} \mathrm{d} x=\sum_{k=1}^{k_{\max }} I\left(t, s_{k}\right) \Delta s=|D| \sum_{k=1}^{k_{\max }} M^{*}\left(t, p_{k}\right) \Delta s=|D| \sum_{k=1}^{k_{\max }} \sum_{\alpha_{k}} b_{\alpha_{k}} M_{\alpha_{k}}^{*} \Delta s .
$$

Comparing this with (13) gives the condition (14) from which the values of $b_{\alpha}$ can be found.

\subsubsection{Detachment of plasmids at the end of microtubules}

It is assumed that all microtubules end at the same Cartesian grid point denoted by $\mathcal{E}$ and located near the nucleus:

$$
\forall q=1 \ldots N_{\mathcal{T}}, \quad \mathcal{E}_{q}=\mathcal{E} .
$$

The total mass at time $t$ at the ending point of a single microtubule $\mathcal{T}$ is $v_{\mathcal{T}} W(t, \mathcal{E})$. It is not possible to describe precisely the ending disc $D(\mathcal{E})$ on the Cartesian grid, unlike in the continuous case, so the whole quantity is added at the ending point $\mathcal{E}$. In order to preserve the total mass during the passage from a point on a microtubule to a two-dimensional cytoplasmatic domain, the last term in equation $7 \mathrm{a}$ is discretized as follows

$$
\frac{v_{\mathcal{T}}}{|D|} W(\mathcal{E}) \delta_{D(\mathcal{E})}(x) \sim \frac{v_{\mathcal{T}}}{\mathcal{V}_{C}} w_{k_{\max }}
$$

where $w_{k_{\max }}$ is the approximated value of $W(\mathcal{E})$. 


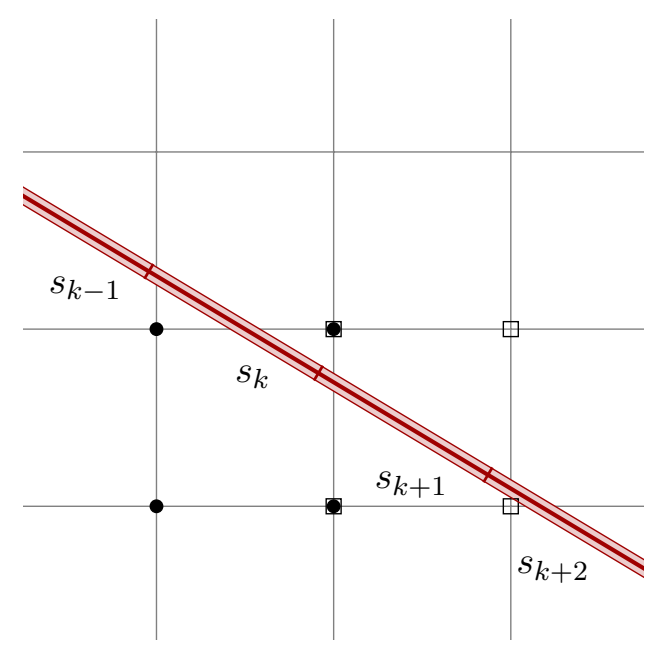

(a) The dimension of the area of attraction is small with respect to the mesh. Only the value at $s_{k}$ is used to compute the integral on $D\left(s_{k}\right)$.

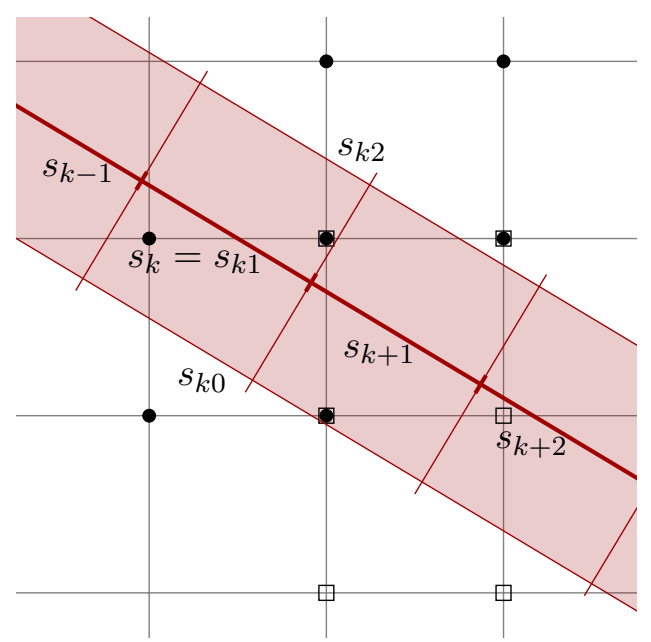

(b) The dimension of the area of attraction is large with respect to the mesh. Three points $s_{k 0}, s_{k 1}, s_{k 2}$ are used to compute the integral on $D\left(s_{k}\right)$.

Figure 2: Discretization of the area of attraction $\mathcal{U}\left(\mathcal{T}_{q}\right)$ of a microtubule on the Cartesian mesh. Computation of the total mass of $M^{*}$ on on a disc $D\left(s_{k}\right)$ involves integration on $m_{\max }$ points $s_{k}^{m}, m=1, \ldots, m_{\max }$. Dark disc $(\bullet)$ represents mesh points used in the interpolation to compute $M^{*}$ at $s_{k}^{m}$, while empty square ( $\square$ ) at $s_{k+1}^{m}$.

\section{Numerical results}

Now we present preliminary simulation results of the transport of DNA plasmids using model (4), (6), (7). It is the qualitative analysis to verify whether the model provides results in agreement with the experimentally observed behavior. The quantity of plasmids that arrive in the vicinity or inside the nucleus is usually one of the main criterion to determine the good model. We study the effect of the active transport along microtubules and the dependence of the accumulation of plasmids in the nucleus on the number of microtubules. Additionally, the influence of different types of electric pulses is considered. However, before these analysis, the validity of the numerical scheme presented in the previous section has to be assessed. In particular, we will control that the total mass of cargo is preserved throughout the simulation, independently from membrane permeabilization and attachment to dynein-importin.

\subsection{Mass conservation}

First we analyze if the numerical scheme, presented in the previous section, preserves the total mass. More precisely, we study the time evolution of the concentration of the plasmid-dynein-importin complex $M^{*}$, given by (7), in a cell without a nucleus and with a single microtubule. $M^{*}$ and $W$ are the only observables considered in this test, and the effect of the electric field is ignored. The domain $\Omega_{h}=[0, L]^{2}$, with $L=30 \mu \mathrm{m}$ is discretized by $J=200$ points in each direction. It contains the cell of radius $R=8 \mu \mathrm{m}$ and the microtubule is discretized by $k_{\max }=50$ nodes. The initial distribution 
$M^{*}(t=0, x)$ of $M^{*}$ is given by

$$
M^{*}(t=0, x)=\exp \left(-\left(4 d_{\Gamma}(x) / R\right)^{2}\right),
$$

where $d_{\Gamma}(x)$ is the distance of $x$ to the membrane and $R$ is the cell radius.

The solutions at time $t=20 \mathrm{~s}$ with and without diffusion are presented in Figure 3(a). One can observe that plasmids attach to the microtubule and are transported towards the center of the cell. As expected, in the absence of diffusion only a small amount of plasmids, which were initially located in the area of attraction of the microtubule, arrive at the center. On the other hand, diffusion brings continously more plasmids within the area of attraction and increase their concentration at the cell center. Figure $3(\mathrm{~b})$ presents the time evolution of the relative error $E_{\mathcal{M}}$ :

$$
E_{\mathcal{M}}(t):=\frac{|\mathcal{M}(t)-\mathcal{M}(t=0)|}{\mathcal{M}(t=0)}
$$

between the total mass of plasmids in the cell at time $t$, given by

$$
\mathcal{M}(t)=\int_{\mathcal{O}_{\mathrm{c}}} M^{*}(x, t) \mathrm{d} x+\int_{\mathcal{T}} W(s, t) \mathrm{d} s \approx \sum_{\mathbf{x}_{i, j} \in \Omega_{h}} M^{*}\left(\mathbf{x}_{i, j}\right) h^{2}+\sum_{k=1}^{k_{\max }} w_{k} \Delta s,
$$

and its initial value. The total mass is conserved with an accuracy of order 2 (the error is of order $10^{-4} \sim \Delta x^{2}$ ) for a diffusion coefficient equal to $d_{\mathrm{c}}^{*}=10^{-14} \mathrm{~m}^{2} / \mathrm{s}$, which is accurate enough. Error may result from the fact that microtubule and cell boundary do not coincide on the mesh grid. Another reason may be the discretization of the Neumann boundary conditions in the diffusion solver due to the approximation of the interface $\Gamma_{\mathrm{c}}$ on the Cartesian mesh. Similar tests were performed in 3D for

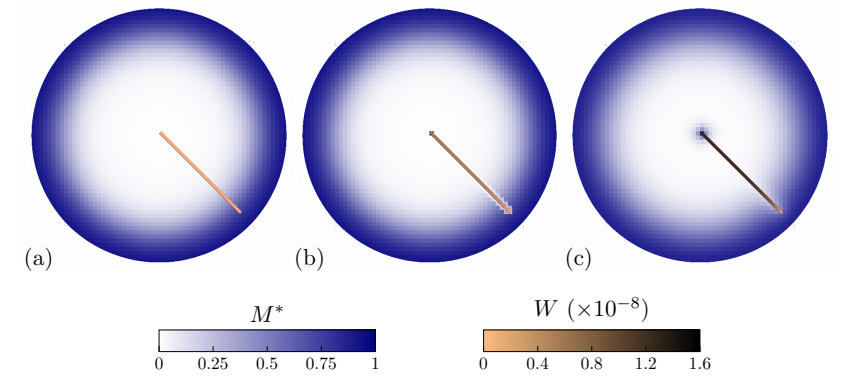

(a) Plasmid concentration in the cell at different time.

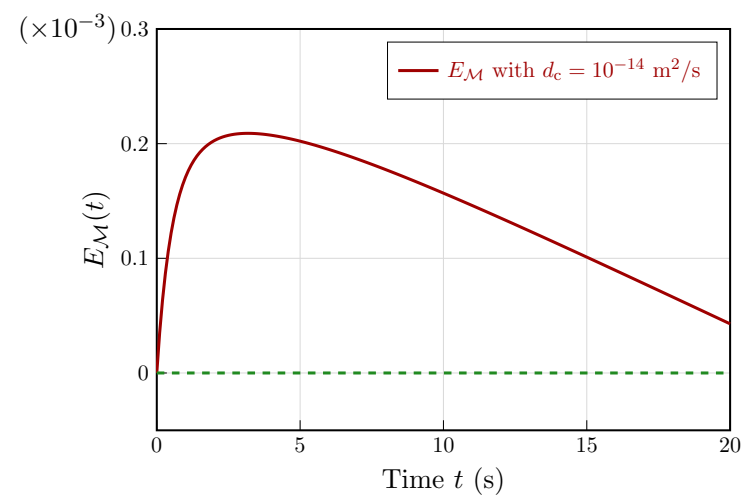

(b) Relative error $E_{\mathcal{M}}$ given by 16 in $2 \mathrm{D}$.

Figure 3: Figure 3(a) (2D): Spatial distribution of the concentration of plasmids in the cytoplasm $M^{*}$ and on the microtubule $W$ : (a) Initial state (b) Result without diffusion at $t=20 \mathrm{~s}$ (c) Result with diffusion $d_{\mathrm{c}}^{*}=10^{-14} \mathrm{~m}^{2} / \mathrm{s}$ at $t=20 \mathrm{~s}$. Figure $3(\mathrm{~b})$ : Relative error of the total mass 16 of the plasmids $M^{*}$ in the cytoplasm with $d_{\mathrm{c}}^{*}=10^{-14}$ $\mathrm{m}^{2} / \mathrm{s}$. Error may result from the fact that microtubule and cell boundary do not coincide on the mesh grid. However the magnitude order of the relative error is about $0.02 \%$, which is accurate. All the numerical constants are given in Table 1

a spherical cell with one microtubule. The computational cost of $3 \mathrm{D}$ simulations prevents from using 
a fine grid $\left(80^{3}\right.$ points are used for the discretization) however one can see that the mass conservation is still well conserved, with a relative error of $0.05 \%$, as presented in Figure 4 .
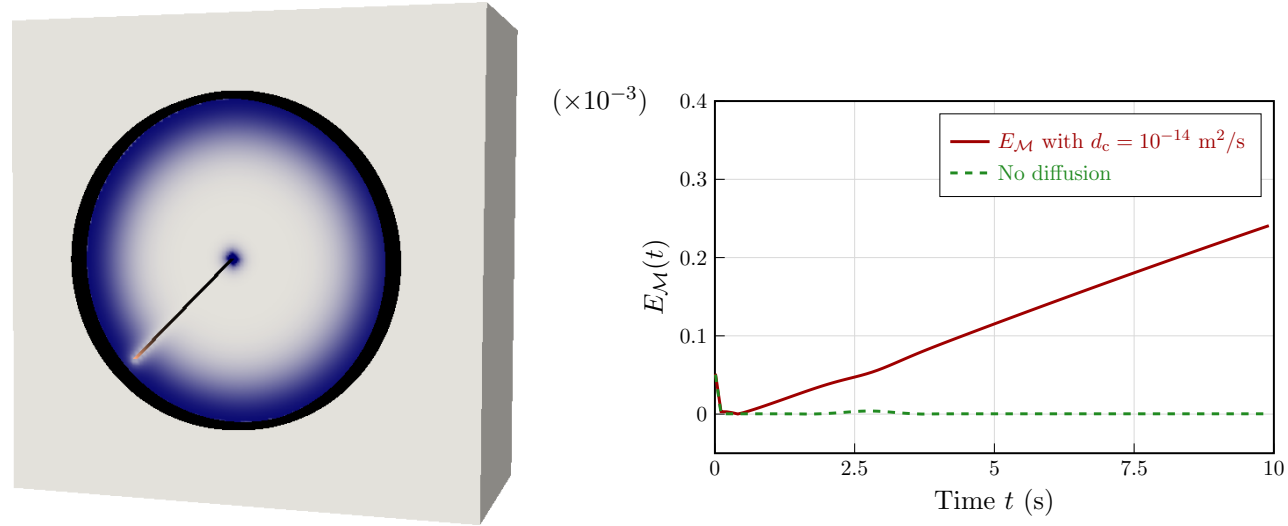

(a) Spatial distribution of the plas- (b) Evolution of the relative error $E_{\mathcal{M}}$ given by (16) in mid concentration in 3D simulation. 3D.

Figure 4: Relative error of the total mass 16 of the plasmids $M^{*}$ in the cytoplasm with $d_{\mathrm{c}}^{*}=10^{-14} \mathrm{~m}^{2} / \mathrm{s}$ in $3 \mathrm{D}$ simulation. The magnitude order of the relative error is about $0.06 \%$, which is very accurate for $3 \mathrm{D}$ simulation. The parameters are given in Table 1] except the number of grid nodes which is $80^{3}$.

\subsection{Effectiveness of the active transport along the microtubules}

For all simulations presented from now on, the computation domain is a circular bi-dimensional cell, with a nucleus and a network of microtubules embedded in the cytoplasm. The position as well as the number of microtubules are arbitrary and does not reproduce any real configuration apart from the fact that they end at the same point near the nucleus. The model and simulation parameters are given in Table 1 unless precised differently.

In this section we present qualitative analysis on the effect of the microtubules on the DNA uptake in the nucleus. We consider a more complex bi-dimensional geometry with respect to works in [23, where qualitative and quantitative results were obtained.

It is worth noting that very recent experimental work of Rosazza et al. [25] and Dean et al. [2] investigated the issue of intra-cellular plasmids transport. These works provides numerous statistics on the trajectories of plasmids aggregates followed by fluorescence and the comparison between transportation of plasmids by free diffusion and by the microtubules. The diffusion coefficients of the plasmids were measured around $10^{-14} \mathrm{~m}^{2} / \mathrm{s}$, which is the value we take for the numerical experiments.

In this test a cell with 17 microtubules is considered. The initial concentration of plasmids $M$ in the extracellular matrix is given by

$$
M(t=0, x)=\mathbb{1}_{\mathcal{O}_{\mathrm{e}}}(x)
$$

and electric pulses of amplitude $V=15 \mathrm{kV} / \mathrm{m}$ and duration $100 \mathrm{~ms}$ are applied. The initial concentration of both plasmids $M$ and cargo $M^{*}$ are set to zero at $t=0$, so that plasmids enter only thanks 
to electropermeabilization.

Spatial distribution of plasmids $M^{*}$ in a cell at time $t=10 \mathrm{~s}$ is presented in Figure 5 . We also monitor the average concentrations of plasmids in the nucleus $\left(\overline{M_{\mathrm{n}}^{*}}\right)$ and in the whole intracellular medium $\left(\overline{M_{\mathrm{cn}}^{*}}\right)$ at each iteration time, as shown in figure 6 . They are computed using the following definitions:

$$
\begin{aligned}
& \overline{M_{\mathrm{n}}^{*}}=\frac{1}{\mathcal{A}_{\mathcal{O}_{\mathrm{n}}}} \sum_{i, j} M^{*}(\mathbf{x} i, j), \\
& \overline{M_{\mathrm{cn}}^{*}}=\frac{1}{\mathcal{A}_{\mathcal{O}_{\mathrm{c}}}+\mathcal{A}_{\mathcal{O}_{\mathrm{n}}}} \sum_{i, j} M^{*}(\mathbf{x} i, j),
\end{aligned}
$$

where $\mathcal{A}_{\mathcal{O}_{\mathrm{c}}}$ and $\mathcal{A}_{\mathcal{O}_{\mathrm{n}}}$ designate the numerical area of $\mathcal{O}_{\mathrm{c}}$ and $\mathcal{O}_{\mathrm{n}}$ respectively.

As observed already in numerical experiments in [23, plasmids concentration near the nucleus increases significantly only in the presence of microtubules. Diffusion alone is incapable of transporting DNA from the cell boundary to its center. The average concentration in the cytoplasm increases in time in both cases due to the inflow from the extracellular matrix. The increase is faster in a cell with microtubules, because the concentration of plasmids on the inner side of the cell boundary decreases faster, and thus the flow through the membrane is higher. The accumulation of plasmids in the nucleus is negligible in a cell without microtubules with respect to the cell with 17 microtubules.
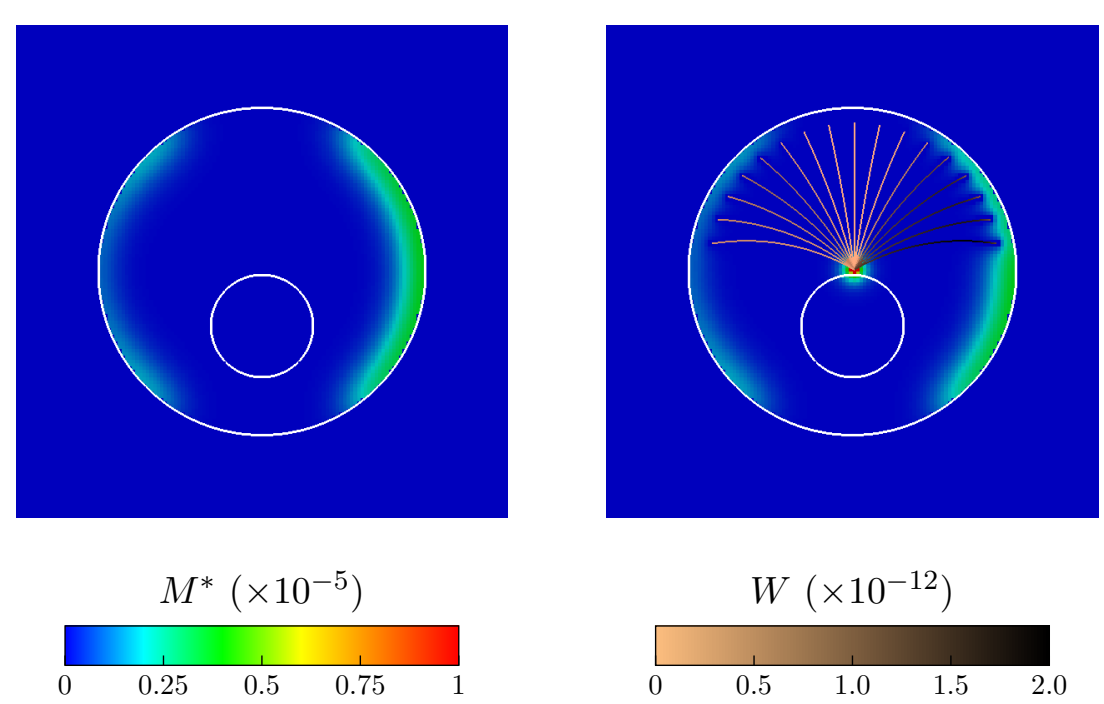

Figure 5: Spatial distribution of the concentration of plasmids $M^{*}$ in a cell without microtubules (on the left) and with 17 microtubules (on the right) at time $t=10 \mathrm{~s}$ for model (6), (7), 8) with the initial data (17) and electric pulses of amplitude $V=15 \mathrm{kV} / \mathrm{m}$ and duration $100 \mathrm{~ms}$.

\subsection{Comparison between high-voltage and low-voltage pulses}

Studies on electroporation shows the influence of the amplitude of the applied voltage and the duration of pulses on the transport of molecules through the cells membrane. In the following simulation 


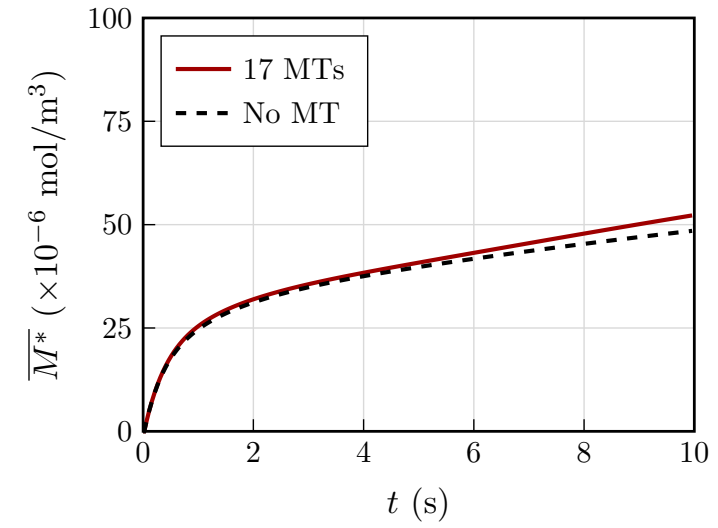

(a) Average plasmid concentration in the cytoplasm.

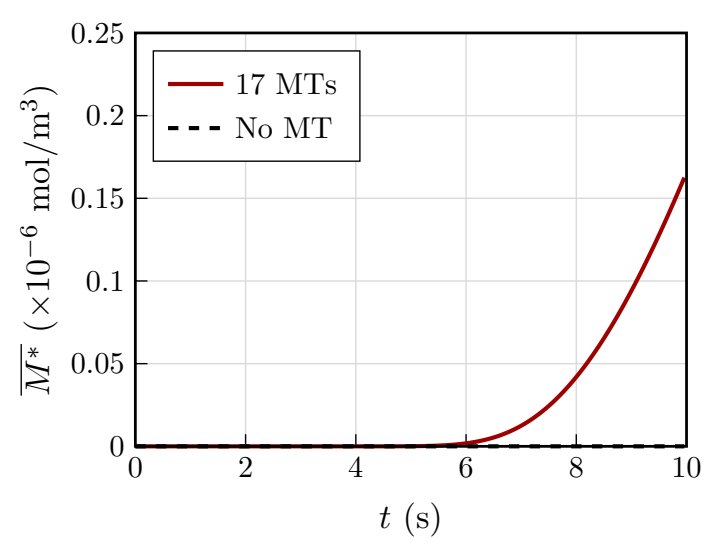

(b) Average plasmid concentration in the nucleus.

Figure 6: Effectiveness of the microtubule network for plasmid uptake in the nucleus. Time evolution of the average concentration of plasmids $M^{*}$ in the cytoplasm (Figure 6(a) and in the nucleus (Figure 6(b) for model (6), (7), (8) with the initial data (17) and electric pulses of amplitude $V=15 \mathrm{kV} / \mathrm{m}$ and duration $100 \mathrm{~ms}$ in case without microtubules (dashed line) and with 17 microtubules (continuous line). The DNA degradation by DNA-ases enzymes prevent the passive diffusion of the DNA up to nucleus: microtubules are necessary to the transfection.

we analyze the effect of different pulses parameters on the accumulation of plasmids in the nucleus after application of the pulses. The three types of pulses, close to electropermeabilization standards, are chosen:

- Pulse A: $40 \mathrm{kV} / \mathrm{m}$ pulse of duration $100 \mu \mathrm{s}$,

- Pulse B: $10 \mathrm{kV} / \mathrm{m}$ pulse of duration $20 \mathrm{~ms}$,

- Pulse C: $1 \mathrm{kV} / \mathrm{m}$ pulse of duration $20 \mathrm{~ms}$.

The same set of parameters as for the previous sections and a cell with 15 microtubules are used. Figure 7) shows even though the membrane conductance during the pulse is quite similar for pulse A and B, milli-pulse B is more efficient than micro-pulse A in terms of concentration of plasmids in the nucleus. The average conductance of the membrane during the pulse is larger in the case of pulse $\mathrm{A}$ then in the case of pulse B $\left(5.4 \mathrm{~S} / \mathrm{cm}^{2}\right.$ vs $\left.0.38 \mathrm{~S} / \mathrm{cm}^{2}\right)$, but the latter lasts much longer, leading to more defects in the membrane. As a result the averaged values of $P_{\mathrm{c}}$ just after the end of pulses $\mathrm{A}$ and $\mathrm{B}$ are respectively $0.74 \times 10^{-10}$ and $2.6 \times 10^{-10} \mathrm{~s}^{-1}$. It explains the difference in the final concentrations of plasmids in the nucleus. The amplitude of the pulse $\mathrm{C}$ is too low to permeabilize the membrane and no plasmids are observed in the nucleus. These results are in agreement with experimental observations showing that low-voltage long pulses (several milliseconds) are necessary to increase the effectiveness of DNA uptakes by cells [1].

\subsection{Influence of the density of microtubules}

In the numerical simulations the geometry of the microtubule network was set arbitrarily, in terms of density and location. In this test we analyze how the number of microtubules in the same region 


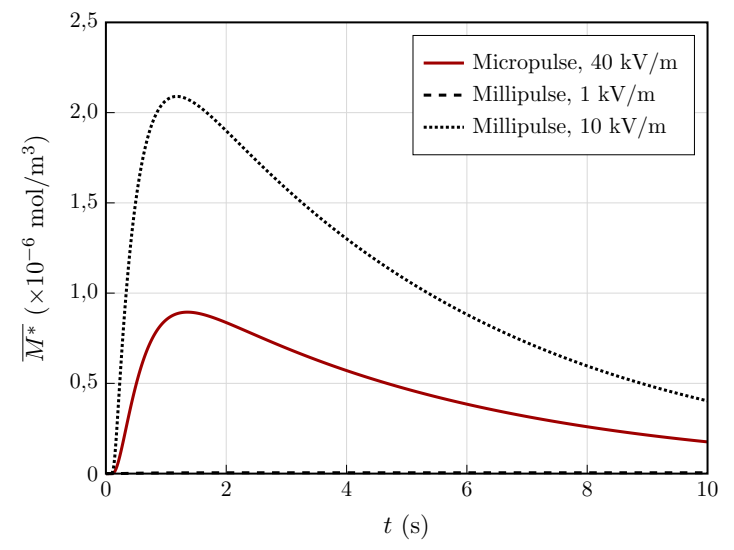

Figure 7: Averaged concentration of plasmids $M^{*}$ in the nucleus for different types of electric pulses. The average membrane conductance and permeability after the pulse A (micropulse) are $S_{\mathrm{c}}=5.4 \mathrm{~S} / \mathrm{cm}^{2}, P_{\mathrm{c}}=7.92 \cdot 10^{-11} \mathrm{~m} / \mathrm{s}$, for pulse B $(10 \mathrm{kV} / \mathrm{m}$ during $20 \mathrm{~ms}) S_{\mathrm{c}}=0.38 \mathrm{~S} / \mathrm{cm}^{2}, P_{\mathrm{c}}=2.59 \cdot 10^{-10} \mathrm{~m} / \mathrm{s}$, and for the pulse C $(1 \mathrm{kV} / \mathrm{m} \mathrm{during} 20 \mathrm{~ms})$, $S_{\mathrm{c}}=S_{0}=1.9 \cdot 10^{-4} \mathrm{~S} / \mathrm{cm}^{2}, P_{\mathrm{c}}=3.36 \cdot 10^{-13} \mathrm{~m} / \mathrm{s}$.

influence the quantity of plasmids in the nucleus. Figure 8(a) shows some examples of simulation setups. Average concentration of cargo in the nucleus is presented in Figure 8(b), Saturation occurs when a density of around 2 microtubules per micrometer of membrane is reached. Note that the density corresponding to overlapping regions of attraction $\mathcal{U}\left(\mathcal{T}_{q}\right)$ right below the membrane is around 50 microtubules per micrometer.

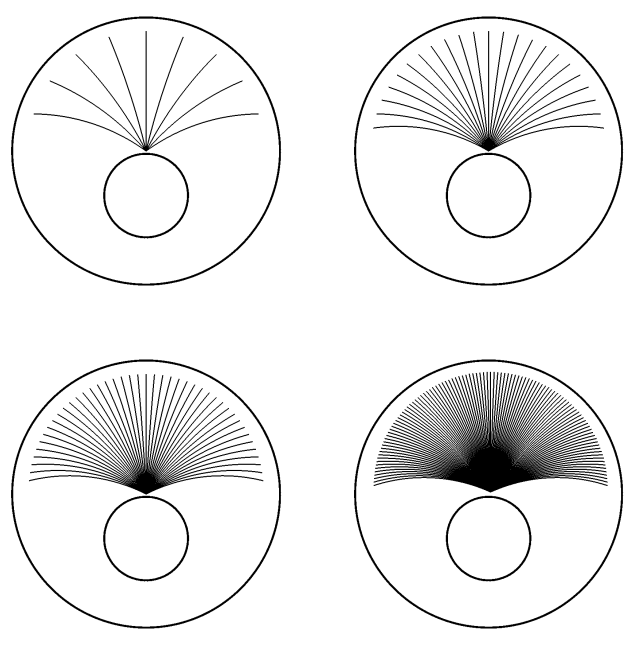

(a) Examples of microtubule network setups in $2 \mathrm{D}$ for $9,25,45$ and 105 microtubules

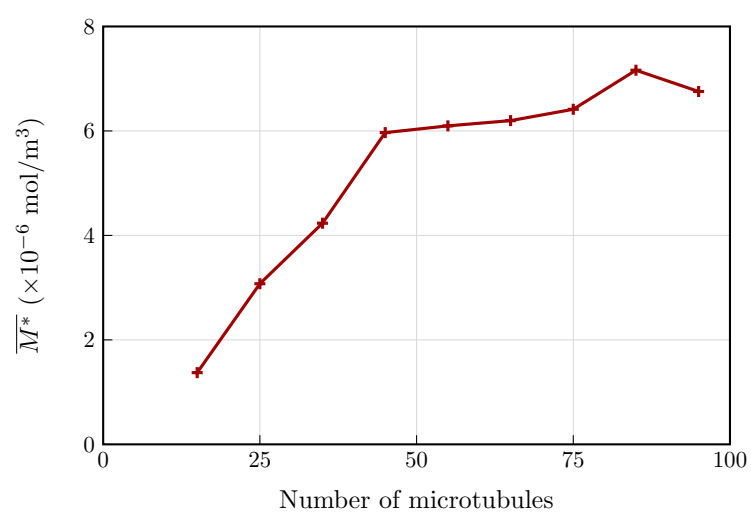

(b) Maximal averaged concentration of cargo $M^{*}$ inside the nucleus as a function of the number of microtubules. 
Table 1: Values of the model parameters.

\begin{tabular}{|c|c|c|c|}
\hline Variable & Symbol & Value & Unit \\
\hline \multicolumn{4}{|l|}{ Cell parameters : } \\
\hline Cell radius & $R$ & 8 & $\mu \mathrm{m}$ \\
\hline Nucleus radius & $R_{\mathrm{n}}$ & 2 & $\mu \mathrm{m}$ \\
\hline Cytoplasmic conductivity & $\sigma_{\mathrm{c}}=\sigma_{\mathrm{n}}$ & 0,455 & $\mathrm{~S} / \mathrm{m}$ \\
\hline Extracellular conductivity & $\sigma_{\mathrm{e}}$ & 1,5 & $\mathrm{~S} / \mathrm{m}$ \\
\hline Membrane capacitance & $C_{\mathrm{c}}=C_{\mathrm{n}}$ & 0,0095 & $\mathrm{~F} / \mathrm{m}^{2}$ \\
\hline Rest surface conductivity & $S_{0}$ & 1,9 & $\mathrm{~S} / \mathrm{m}^{2}$ \\
\hline Nuclear membrane permeability to plasmids & $P_{\mathrm{n}}$ & $10^{-5}$ & $\mathrm{~m} / \mathrm{s}$ \\
\hline \multicolumn{4}{|l|}{ Diffusion parameters : } \\
\hline Extracellular diffusion coefficient of $M$ & $d_{\mathrm{e}}$ & $10^{-11}$ & $\mathrm{~m}^{2} / \mathrm{s}$ \\
\hline Cytoplasmic diffusion coefficient of $M$ & $d_{\mathrm{c}}=d_{\mathrm{n}}$ & $5.10^{-13}$ & $\mathrm{~m}^{2} / \mathrm{s}$ \\
\hline Cytoplasmic diffusion coefficient of $M^{*}$ & $d^{*}$ & $5.10^{-13}$ & $\mathrm{~m}^{2} / \mathrm{s}$ \\
\hline Extracellular motility of $M$ & $\mu_{\mathrm{e}}$ & $1.10^{-7}$ & $\mathrm{mV}^{-1} \mathrm{~s}^{-1}$ \\
\hline \multicolumn{4}{|l|}{ Electroporation model : } \\
\hline Permeabilization threshold & $V_{\mathrm{ep}}$ & 0,2 & $\mathrm{~V}$ \\
\hline Membrane conductance at rest & $S_{0}$ & 1 & $\mathrm{~S} / \mathrm{m}^{2}$ \\
\hline Porous membrane conductance & $S_{1}$ & $10^{6}$ & $\mathrm{~S} / \mathrm{m}^{2}$ \\
\hline Altered membrane conductance & $S_{2}$ & 5 & $\mathrm{~S} / \mathrm{m}^{2}$ \\
\hline Porous membrane permeability & $P_{1}$ & $10^{-7}$ & $\mathrm{~m} / \mathrm{s}$ \\
\hline Altered membrane permeability & $P_{2}$ & $10^{-8}$ & $\mathrm{~m} / \mathrm{s}$ \\
\hline Characteristic time for lipid alteration & $\tau_{+}$ & $10^{-3}$ & $\mathrm{~s}$ \\
\hline Characteristic time for lipid recovery & $\tau_{-}$ & 300 & s \\
\hline \multicolumn{4}{|l|}{ Microtubules parameters : } \\
\hline Length & $\ell_{\mathcal{M}}$ & from 5 to 8 & $\mu \mathrm{m}$ \\
\hline Transport velocity & $v_{\mathcal{T}}$ & 1 & $\mu \mathrm{m} / \mathrm{s}$ \\
\hline Radius of the area of attraction & $r_{0}$ & 20 & $\mathrm{~nm}$ \\
\hline Plasmid-microtubule binding rate & $k_{\mathcal{T}}$ & $10^{6}$ & $\mathrm{~s}^{-1}$ \\
\hline Plasmid-dynein-importin binding rate & $k^{*}$ & 0,5 & $\mathrm{~s}^{-1}$ \\
\hline \multicolumn{4}{|l|}{ Numerical parameters : } \\
\hline Simulation box size & $L$ & 30 & $\mu \mathrm{m}$ \\
\hline Number of grid nodes & $J^{2}$ & $150 \times 150$ & \\
\hline Number of nodes on microtubules & $k_{\max }$ & 50 & \\
\hline Initial concentration of $M$ in $\mathcal{O}_{\mathrm{e}}$ & $M^{0}$ & 1 & $\mathrm{~mol} / \mathrm{m}^{3}$ \\
\hline Time step during the pulse & $\Delta t$ & 10 & $\mu \mathrm{s}$ \\
\hline Time step after the pulse & $\Delta t$ & 10 & $\mathrm{~ms}$ \\
\hline
\end{tabular}

\section{Conclusions}

In this paper we developed a mathematical model describing the transport of DNA plasmids from the extracellular medium up to the nucleus. It couples an electropermeabilization model with a few parameters with the intracellular dynamics system for diffusion of plasmids and their active transport by microtubules network. Numerical schemes, efficient in 2D and 3D, are described, making possible computations in realistic configurations. Note that the complexity of the problem is extremely high and the biological phenomena of the plasmids trafficking are still not completely understood. Nevertheless the proposed model is qualitatively in agreement with experimental data. In particular, using a simple model of a circular cell with a microtubules network of arbitrary shape and location, we showed that the active transport along microtubules is necessary to observe a significant accumulation of plasmids in the nucleus. Additionally, our choice to couple the intracellular transport with the electroporation 
phenomena gave us the possibility to study the influence of the electrical pulses parameters on the transport of plasmids through the cell membrane and subsequently on their quantity in the nucleus.

DNA reptation model for the crossing of the cell membrane has been addressed by De Gennes in the late 90's [6], but the article focused on the membrane crossing of one DNA molecule, and it did not account for the space distribution of DNA in the bulk, neither on the directionality of the electric field, as in the experiments. Extended model has recently been proposed by Dai et al. [5], but here again the focus is performed of the local membrane crossing: neither the anisotropic distribution of DNA nor the DNA transport inside the cell has been addressed. About the electroporation models, as developed by Weaver's group [30] and reference therein, the inner transport of DNA was not accounted for, and they mostly focus on small diffusive molecules, while DNA are mainly driven by electrophoretic forces in the bulk, and active microtubular transport inside the cell. Thus, as far as we know, the overview of the complete DNA transport proposed in this paper has not been addressed before. For calibration purpose, we derive a phenomenological, with as less parameter as possible, but which can account for the main phenomena involved in DNA transfection. Therefore, once the calibration has been performed in specific experiments, one can expect a predictability of the model, for specific configurations. However new experiments dedicated to this calibration has to be performed. This is the second forthcoming step of our current research.

The long-term goal is to propose optimization strategies for DNA transfection. Assuming the predictability of the model, which has to be confirmed by dedicated experiments, the optimization could be performed on the pulse delivery, for a given specific DNA plasmids, or on the DNA plasmids properties, for given electric pulses. In particular, the current hot topic in bioengineering is to provide plasmids with high potentiality of gene expression. We are confident that our model can provide the best compromise to be obtained between the plasmid diffusion property, the affinity with importin for the transport along the microtubule and the degradation properties. It can thus give hints to bioengineers for the plasmid creation.

On the other hand, pulse generators are currently beeing developed to deliver very short (a few nanoseconds long) and high voltage pulse (several $\mathrm{kV} / \mathrm{cm}$ ). According to our model, such pulses alone cannot make the DNA cross the membrane, since electrophoretic forces are needed: optimizing the combination of high and low voltage pulses for the gene transfection, accounting for the DNA degradation is achievable with our model. The very long-term aim is to construct a high potential mathematical tool for therapeutic purposes and possible strategies investigations.

\section{Acknowledgement}

This study has been carried out with financial support from the French State, managed by the French National Research Agency (ANR) in the frame of the "Investments for the future" Programme IdEx Bordeaux - CPU (ANR-10-IDEX-03-02).

Numerical simulations presented in this paper were carried out using the PLAFRIM experimental testbed, being developed under the Inria PlaFRIM development action with support from LABRI and IMB and other entities: Conseil Régional d'Aquitaine, FeDER, Université de Bordeaux and CNRS (see https://plafrim.bordeaux.inria.fr/).

M.L. and C.P. was partly granted by the project Memove (ANR 2011 BS01 006 01). C.P. is also granted by Plan Cancer DYNAMO (Inserm 9749) and Plan Cancer NUMEP (Inserm 11099). 
C.P. is member of LIA EBAM dedicated to electroporation.

[1] F.M. André and J. Gehl et al. Efficiency of high - and low - voltage pulse combinations for gene electrotransfert in mucle, liver tumor and skin. Human Gene Therapy, 19, 2008.

[2] M.A. Badding, E.E. Vaughan, and D.A. Dean. Transcription factor plasmid binding modulates microtubule interactions and intracellular trafficking during gene transfer. Gene therapy, 19(3):338$346,2012$.

[3] A. Cangiani and R. Natalini. A spatial model of cellular molecular trafficking including active transport along microtubules. Journal of theoretical biology, 267(4):614-625, 2010.

[4] M. Cisternino and L. Weynans. A parallel second order cartesian method for elliptic interface problems. $C i C P, 12,2012$.

[5] L. Dai, J. Van Der Maarel, and P.S. Doyle. Extended de gennes regime of dna confined in a nanochannel. A.C.S Publications, Macromolecules, 47, 2014.

[6] P.G. De Gennes. Passive entry of a dna molecule into a small pore. Proc. Natl. Acad. Sci., Bioelectrochemistry, 96, 1999.

[7] K. DeBruin and W. Krassowska. Modelling electroporation in a single cell. I. Effects of field strength and rest potential. Biophys. J., 77(3):1213-1224, 1999.

[8] L. Dimitrio, J. Clairambault, and R. Natalini. A spatial physiological model for p53 intracellular dynamics. Journal of theoretical biology, 316:9-24, 2013.

[9] L. Dimitrio, R. Natalini, and L. Milanesi. A mathematical model for the enhanced cytoplasmic transport - how to get (faster) to the nucleus. In Marco Pellegrini, Ana L. N. Fred, Joaquim Filipe, and Hugo Gamboa, editors, BIOINFORMATICS, pages 39-46. SciTePress, 2011.

[10] A.-T. Dinh, T. Theofanous, and S. Mitragotri. Modeling of pattern regulation in melanophores. Journal of theoretical biology, 244(1):141-153, 2007.

[11] A.-T. Dinh, T.o Theofanous, and S. Mitragotri. A model for intracellular trafficking of adenoviral vectors. Biophysical journal, 89(3):1574-1588, 2005.

[12] J.-M. Escoffre, T. Portet, L. Wasungu, J. Teissié, D. Dean, and M.-P. Rols. What is (still not) known of the mechanism by which electroporation mediates gene transfer and expression in cells and tissues. Molecular biotechnology, 41(3):286-295, 2009.

[13] D. Görlich, M. J Seewald, and K. Ribbeck. Characterization of ran-driven cargo transport and the rangtpase system by kinetic measurements and computer simulation. The EMBO journal, 22(5):1088-1100, 2003.

[14] A. Ivorra, J. Villemejane, and L.M. Mir. Electrical modeling of the influence of medium conductivity on electroporation. Physical Chemistry Chemical Physics, 12(34):10055-10064, 2010. 
[15] O. Kavian, M. Leguèbe, C. Poignard, and L. Weynans. "Classical" Electropermeabilization Modeling at the Cell Scale. J. Math. Biol., 68(1-2):235-265, 2014.

[16] W. Krassowska and P.D. Filev. Modeling electroporation in a single cell. Biophys. J, 92, 2007.

[17] M. Leguèbe, C. Poignard, and L. Weynans. A second-order Cartesian method for the simulation of electropermeabilization cell models. Journal of Computational Physics, 292:114-140, 2015.

[18] M. Leguèbe, A. Silve, L.M. Mir, and C. Poignard. Conducting and permeable states of cell membrane submitted to high voltage pulses. Mathematical and numerical studies validated by the experiments. J. Theor. Biol., 360:83-94, 2014.

[19] N. I. Markevich, M. A. Tsyganov, J. B Hoek, and B. N. Kholodenko. Long-range signaling by phosphoprotein waves arising from bistability in protein kinase cascades. Molecular systems biology, 2(1), 2006.

[20] T. Naka, M. Hatakeyama, N. Sakamoto, and A. Konagaya. Compensation effect of the mapk cascade on formation of phospho-protein gradients. BioSystems, 83(2):167-177, 2006.

[21] J. Neu and W. Krassowska. Asymptotic model of electroporation. Phys. Rev. E, 53(3):3471-3482, 1999.

[22] M.-G. Notarangelo, R. Natalini, and E. Signori. Gene therapy: The role of cytoskeleton in gene transfer studies based on biology and mathematics. Curr Gene Ther, Mar 2014.

[23] Maria Grazia Notarangelo. A Mathematical model of intracellular transport: role of microtubules and Ran cycle in the nucleocytoplasmic plasmids transport in gene therapy anti-tumor. Thesis, Universitá degli studi di Roma, Sapienza, October 2014.

[24] C. Poignard. About the transmembrane voltage potential of a biological cell in time-harmonic regime. ESAIM: Proceedings, 26:162-179, 2009.

[25] C. Rosazza, A. Buntz, T. Rieß, D. Wöll, A. Zumbusch, and M.P. Rols. Intracellular Tracking of Single-plasmid DNA Particles After Delivery by Electroporation. Molecular Therapy, 21(12):22172226, 2013.

[26] R. Seger and E. G. Krebs. The mapk signaling cascade. The FASEB journal, 9(9):726-735, 1995.

[27] E. Signori, S. Iurescia, E. Massi, D. Fioretti, P. Chiarella, M. De Robertis, M. Rinaldi, G. Tonon, and V. M. Fazio. DNA vaccination strategies for anti-tumour effective gene therapy protocols. Cancer Immunology, Immunotherapy, 59(10):1583-1591, October 2010.

[28] A. E. Smith, B. M. Slepchenko, J. C. Schaff, L. M. Loew, and I. G. Macara. Systems analysis of ran transport. Science, 295(5554):488-491, 2002.

[29] D.A. Smith and R.M. Simmons. Models of motor-assisted transport of intracellular particles. Biophysical Journal, 80(1):45-68, 2001. 
[30] K.C. Smith and J.C. Weaver. Electrodiffusion of molecules in aqueous media: a robust, discretized description for electroporation and other transport phenomena. IEEE T. Bio-med. Eng., 59(6):1514-1522, 2012.

[31] M. Sturrock, A. J Terry, D. P Xirodimas, A. M Thompson, and M. A.J. Chaplain. Spatio-temporal modelling of the hes1 and p53-mdm2 intracellular signalling pathways. Journal of theoretical biology, 273(1):15-31, 2011.

[32] M. Sturrock, A. J Terry, D. P Xirodimas, A. M Thompson, and M. A.J. Chaplain. Influence of the nuclear membrane, active transport, and cell shape on the hes1 and p53-mdm2 pathways: insights from spatio-temporal modelling. Bulletin of mathematical biology, 74(7):1531-1579, 2012.

[33] J.C Weaver. Electroporation of cells and tissues. IEEE Trans. on Plasma Sci., 28, 2000.

[34] L. Wegner, W. Frey, and A. Silve. Electroporation of dc-3f cells is a dual process. Biophys. J, 108, 2015.

\section{Appendix: Proof of Theorem 2.2}

This appendix section is devoted to the sketch of the proof of Theorem 2.2. The well-posedness of problem (4) can be tackled very similarly to Theorem 10, pp251 [15]. The difference here lies in the third domain $\mathcal{O}_{n}$. The main idea of the proof consists in rewriting problem (4) as an equivalent problem, whose unknowns are defined on the surfaces $\Gamma_{\mathrm{c}}$ and $\Gamma_{\mathrm{n}}$ thanks to standard Steklov-Poincaré operators (also called Dirichlet-to-Neumann maps).

More precisely, we define 7 maps $\mathcal{A}_{o}, \mathcal{A}_{\mathrm{c}}, \mathcal{B}_{\mathrm{c}}, \mathcal{B}_{\mathrm{n}}, \mathcal{C}_{\mathrm{c}}, \mathcal{C}_{\mathrm{n}}$ and $\Lambda_{\mathrm{n}}$ as follows:

- $\forall(f, g) \in H^{1 / 2}\left(\Gamma_{\mathrm{D}}\right) \times H^{1 / 2}\left(\Gamma_{\mathrm{c}}\right), \quad \mathcal{A}_{o}(f):=-\left.\sigma_{\mathrm{e}} \partial_{\boldsymbol{\nu}} u\right|_{\Gamma_{\mathrm{c}}}, \quad \mathcal{A}_{\mathrm{c}}(g):=-\left.\sigma_{\mathrm{e}} \partial_{\boldsymbol{\nu}} v\right|_{\Gamma_{\mathrm{c}}}$

where $(u, v)$ are given by

$\left\{\begin{array}{l}\Delta u=0, \quad \text { in } \mathcal{O}_{\mathrm{e}}, \\ \left.u\right|_{\Gamma_{\mathrm{c}}}=0,\left.\quad u\right|_{\Gamma_{\mathrm{D}}}=f,\left.\quad \partial_{\boldsymbol{\nu}} u\right|_{\Gamma_{\mathrm{N}}}=0,\end{array} \quad \begin{cases}\Delta v=0, & \text { in } \mathcal{O}_{\mathrm{e}}, \\ \left.v\right|_{\Gamma_{\mathrm{c}}}=g, & \left.u\right|_{\Gamma_{\mathrm{D}}}=0,\left.\quad \partial \boldsymbol{\nu} v\right|_{\Gamma_{\mathrm{N}}}=0,\end{cases}\right.$

- $\forall(f, g) \in H^{1 / 2}\left(\Gamma_{\mathrm{c}}\right) \times H^{1 / 2}\left(\Gamma_{\mathrm{n}}\right), \quad \mathcal{B}_{\mathrm{c}}(f):=\left.\sigma_{\mathrm{c}} \partial_{\boldsymbol{\nu}} u\right|_{\Gamma_{\mathrm{c}}}, \quad \mathcal{B}_{\mathrm{n}}(g):=\left.\sigma_{\mathrm{c}} \partial_{\boldsymbol{\nu}} v\right|_{\Gamma_{\mathrm{c}}}$

where $(u, v)$ are given by

$$
\left\{\begin{array} { l l } 
{ \Delta u = 0 , } & { \text { in } \mathcal { O } _ { \mathrm { c } } , } \\
{ u | _ { \Gamma _ { \mathrm { c } } } = f , } & { u | _ { \Gamma _ { \mathrm { n } } } = 0 , }
\end{array} \quad \left\{\begin{array}{ll}
\Delta v=0, & \text { in } \mathcal{O}_{\mathrm{c}} \\
\left.v\right|_{\Gamma_{\mathrm{c}}}=0, & \left.v\right|_{\Gamma_{\mathrm{n}}}=g
\end{array}\right.\right.
$$

- $\forall(f, g) \in H^{1 / 2}\left(\Gamma_{\mathrm{c}}\right) \times H^{1 / 2}\left(\Gamma_{\mathrm{n}}\right), \quad \mathcal{C}_{\mathrm{c}}(f):=-\left.\sigma_{\mathrm{c}} \partial_{\nu} u\right|_{\Gamma_{\mathrm{n}}}, \quad \mathcal{C}_{\mathrm{n}}(g):=-\left.\sigma_{\mathrm{c}} \partial_{\boldsymbol{\nu}} v\right|_{\Gamma_{\mathrm{n}}}$ 
where $(u, v)$ are given by

$$
\left\{\begin{array} { l l } 
{ \Delta u = 0 , } & { \text { in } \mathcal { O } _ { \mathrm { c } } , } \\
{ u | _ { \Gamma _ { \mathrm { c } } } = f , } & { u | _ { \Gamma _ { \mathrm { n } } } = 0 , }
\end{array} \quad \left\{\begin{array}{ll}
\Delta v=0, & \text { in } \mathcal{O}_{\mathrm{c}}, \\
V_{\Gamma_{\Gamma_{\mathrm{c}}}}=0, & \left.v\right|_{\Gamma_{\mathrm{n}}}=g,
\end{array}\right.\right.
$$

- $\forall f \in H^{1 / 2}\left(\Gamma_{\mathrm{n}}\right), \quad \Lambda_{\mathrm{n}}(f):=\left.\sigma_{\mathrm{n}} \partial_{\nu} u\right|_{\Gamma_{\mathrm{n}}}$,

where $u$ is given by

$$
\left\{\begin{array}{l}
\Delta u=0, \quad \text { in } \mathcal{O}_{\mathrm{n}}, \\
\left.u\right|_{\Gamma_{\mathrm{n}}}=f .
\end{array}\right.
$$

Note that all these operators, except $\Lambda_{\mathrm{n}}$ are positive and inversible from $H^{1 / 2}(\mathcal{S})$ into $H^{-1 / 2}(\mathcal{S})$, where $\mathcal{S}$ stands either for $\Gamma_{\mathrm{c}}$ or $\Gamma_{\mathrm{n}}$. The operator $\Lambda_{\mathrm{n}}$ is positive and invertible modulo the constant functions. Thanks to these Dirichlet-to-Neumann maps, finding the solution $U$ to problem (4) is equivalent to finding $\left(u_{\mathrm{c}}^{+}, u_{\mathrm{c}}^{-}, u_{\mathrm{n}}^{+}, u_{\mathrm{n}}^{-}\right)$solution to

$$
\begin{array}{cl}
\text { on } \Gamma_{\mathrm{c}}: & \left\{\begin{array}{l}
\mathcal{A}_{o}(g)+\mathcal{A}_{\mathrm{c}}\left(u_{\mathrm{c}}^{+}\right)+\mathcal{B}_{\mathrm{c}}\left(u_{\mathrm{c}}^{-}\right)+\mathcal{B}_{\mathrm{n}}\left(u_{\mathrm{n}}^{+}\right)=0, \\
C_{\mathrm{c}} \partial_{t} V_{\Gamma_{\Gamma_{\mathrm{c}}}}+S_{\mathrm{c}}\left(V_{\Gamma_{\Gamma_{\mathrm{c}}}}\right) V_{\Gamma_{\Gamma_{\mathrm{c}}}}=\mathcal{B}_{\mathrm{c}}\left(u_{\mathrm{c}}^{-}\right)+\mathcal{B}_{\mathrm{n}}\left(u_{\mathrm{n}}^{+}\right),
\end{array}\right. \\
\text {on } \Gamma_{\mathrm{n}}: \quad\left\{\begin{array}{l}
\mathcal{C}_{\mathrm{c}}\left(u_{\mathrm{c}}^{-}\right)+\mathcal{C}_{\mathrm{n}}\left(u_{\mathrm{n}}^{+}\right)+\Lambda_{\mathrm{n}}\left(u_{\mathrm{n}}^{-}\right)=0, \\
C_{\mathrm{n}} \partial_{t} V_{\Gamma_{\mathrm{n}}}+S_{\mathrm{n}} V_{\Gamma_{\Gamma_{\mathrm{n}}}}=\Lambda_{\mathrm{n}}\left(u_{\mathrm{n}}^{-}\right),
\end{array}\right. \\
\text {where } \quad V_{\Gamma_{\Gamma_{\mathrm{c}}}}=u_{\mathrm{c}}^{+}-u_{\mathrm{c}}^{-} \quad \text { and } \quad V_{\Gamma_{\mathrm{n}}}=u_{\mathrm{n}}^{+}-u_{\mathrm{n}}^{-} .
\end{array}
$$

One can pass from problem (4) to problem (23) as follows: The solution $U$ to problem (4) leads to $\left(u_{c}^{+}, u_{c}^{-}, u_{n}^{+}, u_{n}^{-}\right)$solution to (23) thanks to

$$
\left(u_{c}^{+}, u_{c}^{-}, u_{n}^{+}, u_{n}^{-}\right)=\left(\left.U\right|_{\Gamma_{\mathrm{c}}^{+}},\left.U\right|_{\Gamma_{\mathrm{c}}^{-}},\left.U\right|_{\Gamma_{\mathrm{n}}^{+}},\left.U\right|_{\Gamma_{\mathrm{n}}^{-}}\right), \quad V_{\Gamma_{\Gamma_{\mathrm{c}}}}=[U]_{\Gamma_{\mathrm{c}}}, \quad V_{\left.\right|_{\Gamma_{\mathrm{n}}}}=[U]_{\Gamma_{\mathrm{n}}} .
$$

On the other hand, from $\left(u_{c}^{+}, u_{c}^{-}, u_{n}^{+}, u_{n}^{-}\right)$solution to (23), the electric potential $U$ solution to (4) is given as

$$
\left\{\begin{array}{l}
\Delta U=0, \text { in } \mathcal{O}_{\mathrm{e}} \cup \mathcal{O}_{\mathrm{c}} \cup \mathcal{O}_{\mathrm{n}}, \\
U=g(t), \text { on } \Gamma_{\mathrm{D}}, t>0, \\
\partial_{\nu} U=0, \text { on } \Gamma_{\mathrm{N}}, t>0, \\
\left(\left.U\right|_{\Gamma_{\mathrm{c}}^{+}},\left.U\right|_{\Gamma_{\mathrm{c}}^{-}},\left.U\right|_{\Gamma_{\mathrm{n}}^{+}},\left.U\right|_{\Gamma_{\mathrm{n}}^{-}}\right)=\left(u_{c}^{+}, u_{c}^{-}, u_{n}^{+}, u_{n}^{-}\right) .
\end{array}\right.
$$

The key-point of the proof lies in writing problem (23) as a quasilinear evolution system, in order to use the standard semi-group contraction theory. Define the matrix operator $\mathcal{M}$ defined on $H^{1 / 2}\left(\Gamma_{\mathrm{c}}\right) \times$ $H^{1 / 2}\left(\Gamma_{\mathrm{n}}\right)$ into $H^{-1 / 2}\left(\Gamma_{\mathrm{c}}\right) \times H^{-1 / 2}\left(\Gamma_{\mathrm{n}}\right)$ as

$$
\mathcal{M}=\left(\begin{array}{cc}
\mathcal{A}_{\mathrm{c}}+\mathcal{B}_{\mathrm{c}} & \mathcal{B}_{\mathrm{n}} \\
\mathcal{C}_{\mathrm{c}} & \mathcal{C}_{\mathrm{n}}+\Lambda_{\mathrm{n}}
\end{array}\right)
$$


Thanks to the definition of the Dirichlet-to-Neumann maps, one can easily verify that $\mathcal{M}$ is definite positive and thus it is invertible, and its inverse is also positive. Using (23), we infer that

$$
\left(\begin{array}{l}
u_{\mathrm{c}}^{-} \\
u_{\mathrm{n}}^{-}
\end{array}\right)=-\mathcal{M}^{-1}\left(\begin{array}{cc}
\mathcal{A}_{\mathrm{c}} & \mathcal{B}_{\mathrm{n}} \\
0 & \mathcal{C}_{\mathrm{n}}
\end{array}\right)\left(\begin{array}{l}
V_{\Gamma_{\Gamma_{\mathrm{c}}}} \\
V_{\Gamma_{\mathrm{n}}}
\end{array}\right)-\mathcal{M}^{-1}\left(\begin{array}{c}
\mathcal{A}_{o}(g) \\
0
\end{array}\right) \text {. }
$$

Defining the matrix operator $\mathcal{L}$ and the source vector $G:=\left(G_{\mathrm{C}}, G_{\mathrm{n}}\right)^{T}$ as

$$
\begin{aligned}
\mathcal{L} & =\left(\begin{array}{cc}
S_{0} & 0 \\
0 & S_{\mathrm{n}}
\end{array}\right)+\mathcal{M}^{-1}\left(\begin{array}{cc}
\mathcal{A}_{\mathrm{c}} & \mathcal{B}_{\mathrm{n}} \\
0 & \mathcal{C}_{\mathrm{n}}
\end{array}\right), \\
\left(\begin{array}{c}
G_{\mathrm{c}} \\
G_{\mathrm{n}}
\end{array}\right) & =-\mathcal{M}^{-1}\left(\begin{array}{c}
\mathcal{A}_{o}(g) \\
0
\end{array}\right),
\end{aligned}
$$

problem 23) reads as the following quasilinear evolution problem on $\left(V_{\Gamma_{\Gamma_{\mathrm{c}}}}, V_{\Gamma_{\Gamma_{\mathrm{n}}}}\right)^{T}$ :

$$
\begin{aligned}
& \left(\begin{array}{cc}
C_{\mathrm{c}} & 0 \\
0 & C_{\mathrm{n}}
\end{array}\right)\left(\partial_{t}\left(\begin{array}{c}
V_{\left.\right|_{\Gamma_{\mathrm{c}}}} \\
V_{\Gamma_{\mathrm{n}}}
\end{array}\right)\right)+\mathcal{L}\left(\begin{array}{c}
V_{\left.\right|_{\Gamma_{\mathrm{c}}}} \\
V_{\Gamma_{\mathrm{n}}}
\end{array}\right)=\left(\begin{array}{c}
G_{\mathrm{c}} \\
G_{\mathrm{n}}
\end{array}\right)-\left(\begin{array}{c}
\left(S_{\mathrm{c}}\left(V_{\left.\right|_{\Gamma_{\mathrm{c}}}}\right)-S_{0}\right) V_{\Gamma_{\mathrm{c}}} \\
0
\end{array}\right), \\
& \left(\mathcal{A}_{\mathrm{c}}+\mathcal{B}_{\mathrm{c}}\right)\left(u_{\mathrm{c}}^{-}\right)+\mathcal{B}_{\mathrm{n}}\left(u_{\mathrm{n}}^{-}\right)=-\mathcal{A}_{o}(g)-\mathcal{A}_{\mathrm{c}}\left(V_{\Gamma_{\Gamma_{\mathrm{c}}}}\right)-\mathcal{B}_{\mathrm{n}}\left(V_{\Gamma_{\Gamma_{\mathrm{n}}}}\right), \\
& \mathcal{C}_{\mathrm{c}}\left(u_{\mathrm{c}}^{-}\right)+\left(\mathcal{C}_{\mathrm{n}}+\Lambda_{\mathrm{n}}\right)\left(u_{\mathrm{n}}^{-}\right)=-\mathcal{C}_{\mathrm{n}}\left(V_{\left.\right|_{\Gamma_{\mathrm{n}}}}\right) .
\end{aligned}
$$

By definition of the Dirichlet-to-Neumann maps, the operator $\mathcal{L}$ is positive since for any couple of functions $(\varphi, \psi) \in H^{1 / 2}\left(\Gamma_{\mathrm{c}}\right) \times H^{1 / 2}\left(\Gamma_{\mathrm{n}}\right)$

$$
\left\langle\mathcal{L}\left(\begin{array}{c}
\varphi \\
\psi
\end{array}\right) ;\left(\begin{array}{c}
\varphi \\
\psi
\end{array}\right)\right\rangle \geq S_{0} \int_{\Gamma_{\mathrm{c}}}|\varphi|^{2} d x+S_{\mathrm{n}} \int_{\Gamma_{\mathrm{n}}}|\psi|^{2} d x \geq 0 .
$$

Moreover, thanks to standard Lax-Milgram lemma, for any $\lambda>0$ and any $\left(f_{\mathrm{c}}, f_{\mathrm{n}}\right) \in L^{2}\left(\Gamma_{\mathrm{c}}\right) \times L^{2}\left(\Gamma_{\mathrm{n}}\right)$, there exists a unique solution $5 \in P H^{3 / 2}(\Omega)$ to the following problem

$$
\begin{aligned}
\Delta U & =0 \quad \text { in } \mathcal{O}_{\mathrm{e}} \cup \mathcal{O}_{\mathrm{c}} \cup \mathcal{O}_{\mathrm{n}}, \\
\left.\sigma_{\mathrm{e}} \partial_{\boldsymbol{\nu}} U\right|_{\Gamma_{\mathrm{c}}^{+}} & =\left.\sigma_{\mathrm{c}} \partial_{\boldsymbol{\nu}} U\right|_{\Gamma_{\mathrm{c}}^{-}}, \quad S_{0}[U]_{\Gamma_{\mathrm{c}}}-\left.\lambda \sigma_{\mathrm{c}} \partial_{\boldsymbol{\nu}} U\right|_{\Gamma_{\mathrm{c}}^{-}}=f_{\mathrm{c}},
\end{aligned}
$$

and across $\Gamma_{\mathrm{n}}$ :

$$
\left.\sigma_{\mathrm{c}} \partial_{\nu} U\right|_{\Gamma_{\mathrm{n}}^{+}}=\left.\sigma_{\mathrm{n}} \partial_{\nu} U\right|_{\Gamma_{\mathrm{n}}^{-}}, \quad S_{\mathrm{n}}[U]_{\Gamma_{\mathrm{n}}}-\left.\lambda \sigma_{\mathrm{n}} \partial_{\nu} U\right|_{\Gamma_{\mathrm{n}}^{-}}=f_{\mathrm{n}},
$$

and the boundary conditions:

$$
U=0 \quad \text { on } \partial \Omega_{\mathrm{D}}, \quad \partial_{\nu} U=0 \quad \text { on } \partial \Omega_{\mathrm{N}},
$$

and thus the operator $\left(\mathcal{L}, H^{1}\left(\Gamma_{\mathrm{c}}\right) \times H^{1}\left(\Gamma_{\mathrm{n}}\right)\right.$ is m-accretive, and generates a semi-group of contraction.

Since the function $v \mapsto v S_{\mathrm{c}}(v)$ is clearly smooth, bounded and Lipschitz, the non-linearity of the semi-linear problem is not a difficult issue. The procedure follows the proof of Theorem 10 in Kavian et al. [15].

\footnotetext{
${ }^{5}$ For any $s \geq 0$, the functional space $P H^{s}(\Omega)$ is defined as

$$
P H^{s}(\Omega):=\left\{u \in L^{2}\left(\Omega:\left.u\right|_{\mathcal{O}} \in H^{s}(\mathcal{O}), \text { for } \quad \mathcal{O} \in\left\{\mathcal{O}_{\mathrm{e}}, \mathcal{O}_{\mathrm{c}}, \mathcal{O}_{\mathrm{n}}\right\}\right\} .\right.
$$
}

